\title{
ASYMPTOTICS OF OPERATOR SEMIGROUPS VIA THE SEMIGROUP AT INFINITY
}

\author{
JOCHEN GLÜCK AND MARKUS HAASE \\ Dedicated to Ben de Pagter on the occasion of his 65th birthday.
}

\begin{abstract}
We systematize and generalize recent results of Gerlach and Glück on the strong convergence and spectral theory of bounded (positive) operator semigroups $\left(T_{s}\right)_{s \in S}$ on Banach spaces (lattices). (Here, $S$ can be an arbitrary commutative semigroup, and no topological assumptions neither on $S$ nor on its representation are required.) To this aim, we introduce the "semigroup at infinity" and give useful criteria ensuring that the well-known Jacobs-de Leeuw-Glicksberg splitting theory can be applied to it.

Next, we confine these abstract results to positive semigroups on Banach lattices with a quasi-interior point. In that situation, the said criteria are intimately linked to so-called AM-compact operators (which entail kernel operators and compact operators); and they imply that the original semigroup asymptotically embeds into a compact group of positive invertible operators on an atomic Banach lattice. By means of a structure theorem for such group representations (reminiscent of the Peter-Weyl theorem and its consequences for Banach space representations of compact groups) we are able to establish quite general conditions implying the strong convergence of the original semigroup.

Finally, we show how some classical results of Greiner (1982), Davies (2005), Keicher (2006) and Arendt (2008) and more recent ones by Gerlach and Glück (2017) are covered and extended through our approach.
\end{abstract}

\section{INTRODUCTION}

In this paper we deal with the problem of finding useful criteria for the strong convergence of a bounded operator semigroup $T$ on a Banach space $E$, with a special view on the asymptotics of certain semigroups of positive operators on Banach lattices. This problem is not at all new, but we refrain from even trying to give a list of relevant literature at this point. (However, cf. Section 7 below.) Rather, let us stress some features that distinguish our approach from most others.

Classically, asymptotics of operator semigroups focusses on strongly continuous one-parameter semigroups $T=\left(T_{t}\right)_{t \in[0, \infty)}$. However, there are important instances of operator semigroups which lack strong continuity, e.g., the heat semigroup on the space of bounded continuous functions on $\mathbb{R}$ or on the space of finite measures over $\mathbb{R}$ or, in an abstract context, dual semigroups of $C_{0}$-semigroups on non-reflexive

Date: December 17, 2018.

1991 Mathematics Subject Classification. Primary 47D06; Secondary 20M30, 46B42, 47B34, 47B65, 47D03.

Key words and phrases. Convergence of operator semigroups; Jacobs-de Leeuw-Glicksberg theory; positive semigroup representations; positive group representations; AM-compact operators; kernel operators; triviality of the peripheral point spectrum. 
spaces. (See the recent paper Kun18 for a more involved concrete example.) Hence, there is a need for results on asymptotics beyond $C_{0}$-semigroups.

Secondly, besides the "continuous time" case just mentioned, there is an even more fundamental interest in "discrete time", i.e., in the asymptotics of the powers $T^{n}$ of a single operator $T$. From a systematic point of view, it is desirable to try to cover both cases at the same time as far as possible. This is the reason why we consider general semigroup representations $\left(T_{s}\right)_{s \in S}$-where $(S,+)$ is an Abelian semigroup with zero element 0 - as bounded operators on a Banach space without any further topological assumptions (see Section 2).

It may not come as a surprise that non-trivial results about asymptotics can be obtained - even in such a general setting - by employing the so-called JacobsdeLeeuw-Glicksberg (JdLG) theory for compact (Abelian) semitopological semigroups. In fact, the role of the JdLG-theory for asymptotics is well-established. Usually, it is applied to the semigroup

$$
\mathcal{T}:=\operatorname{cl}\left\{T_{s} \mid s \in S\right\}
$$

(closure in the strong or weak operator topology) and hence rests on a "global" compactness requirement for the whole semigroup. This is appropriate for the abovementioned "classical" cases (powers of a single operator, $C_{0}$-semigroups) because there the strong compactness of $\mathcal{T}$ is necessary for the convergence of $T$. However, typical examples of non-continuous shift semigroups (left shift on $\mathrm{L}^{\infty}(0,1)$ or $\mathrm{c}_{0}\left(\mathbb{R}_{+}\right)$) show that such semigroups may converge strongly to 0 without being relatively strongly compact. (The left shift semigroup on $c_{0}\left(\mathbb{R}_{0}\right)$ is not even eventually relatively strongly compact.)

In order to cover also these more general situations, we introduce the set

$$
\mathcal{T}_{\infty}:=\bigcap_{t \in S} \operatorname{cl}\left\{T_{s+t} \mid s \in S\right\}
$$

which we call the semigroup at infinity. It turns out that $\mathcal{T}_{\infty}$ is a good replacement for $\mathcal{T}$ if $\mathcal{T}_{\infty}$ is strongly compact and not empty. In particular, its minimal projection, $P_{\infty}$, satisfies

$$
P_{\infty} x=0 \quad \Longleftrightarrow \quad \lim _{s \in S} T_{s} x=0 \quad(x \in E)
$$

(see Theorem 2.2). Not surprisingly, in the mentioned "classical" cases the condition that $\mathcal{T}_{\infty}$ be strongly compact and not empty is actually equivalent to relative strong compactness of the original semigroup (cf. Remark 2.6), and hence the use of $\mathcal{T}_{\infty}$ is then - in some sense - unnecessary. In a general appoach going beyond these classical cases, however, it is our means of choice.

In a next step (Section 3), we describe a convenient set-up that warrants the crucial property (i.e.: $\mathcal{T}_{\infty}$ is non-empty and compact). To this end, the new notion of quasi-compactness relative to a subspace is introduced. This condition generalizes the traditional notion of quasi-compactness of a semigroup and plays a central role in our first main result (Theorem 3.1).

After these completely general considerations, and from then on until the end of the paper, we confine our attention to positive operator semigroups on Banach lattices. Our second main result, Theorem 4.3, appears to be a mere instantiation of Theorem 3.1 in such a setting. However, the theorem gains its significance from the fact that the required quasi-compactness condition is intimately linked to the 
well-known property of $A M$-compactness of positive operators which, in turn, occurs frequently when dealing with "concrete" positive semigroups arising in evolution equations and stochastics (see Appendix $\mathrm{A}$ ).

The main thrust of Theorem 4.3 is that it reduces the study of the asymptotic properties of certain positive semigroups to the following special case: $E$ is an atomic Banach lattice and $T$ embeds into a strongly compact group of positive invertible operators thereon. Hence, in the subsequent Section 5 we analyze this situation thoroughly and establish a structure theorem which is reminiscent of the Peter-Weyl theorem and its consequences for Banach space representations of compact groups (Theorem 5.4).

Putting the pieces together, in Section 6 we formulate several consequences regarding the asymptotic (and spectral-theoretic) properties of a positive semigroup $T=\left(T_{s}\right)_{s \in S}$ satisfying the conditions of Theorem 4.3. Particularly important here is the fact that we can identify (in Theorem 6.2) two intrinsic properties of the semigroup $S$ that imply the convergence of $T$ : an algebraic one (essential divisibility of $S$ ) and a topological one (under the condition that $S$ is topological and $T$ is continuous). Interestingly enough, both are applicable in the case $S=\mathbb{R}_{+}$, but none of them in the case $S=\mathbb{N}_{0}$.

Finally, in Section 7 we review the pre-history of the problem and show how the results obtained so far by other people relate to (or are covered by) our findings. Interestingly, in this pre-history the spectral-theoretic results ("triviality of the point spectrum") have taken a much more prominent role than the asymptotic results. We end the paper with a new and unifying result in this direction (Theorem 7.6).

Relevance and Relation to the Work of Others. The present work can be understood as a continuation and further development of the recent paper GG17b] by M. Gerlach and the first author. Many ideas in the present paper can already be found in GG17b, like that one can go beyond strong continuity by combining the JdLG-theory with the concept of AM-compactness; or that under AM-compactness conditions a purely algebraic property (divisibility) of the underlying semigroup suffices to guarantee the strong convergence of the representation.

However, we surpass our reference in many respects:

1) A general Banach space principle (Theorem 3.1) is established and identified as the theoretical core which underlies the results of GG17b. This principle, which is based on the new notion of "quasi-compactness relative to a subspace" and on our systematic study of the "semigroup at infinity" (Theorem 2.2), has potential applications in the asymptotic theory of semigroups without any positivity assumptions.

2) As a consequence of 1), the two main results from GG17b, Theorem 3.7 and Theorem 3.11, are now unified. Moreover, our results hold without requiring the semigroup to have a quasi-interior fixed point.

3) A general structure theorem for representations of compact groups on atomic Banach lattices (Theorem 5.4) is established. This result is auxiliary to-but actually completely independent of - our principal enterprise, the asymptotics of operator semigroups. From a different viewpoint, it is a contribution to 
the theory of positive group representations as promoted by Marcel de Jeu (Leiden) and his collaborators.

4) A new spectral-theoretic result (Theorem [7.6) about the properties of unimodular eigenvalues is establied.

To understand the relevance of the results obtained in this paper, one best looks into the pre-history of its predecessor GG17b. We decided to place such a historical narrative after our systematic considerations, in Section 7 . That gives us the possibility to then refer freely to the results proven before, and to explain in detail their relation to the results obtained earlier by other people.

Notation and Terminology. We use the letters $E, F, \ldots$ generically to denote Banach spaces or Banach lattices over the scalar field $\mathbb{K} \in\{\mathbb{R}, \mathbb{C}\}$. The space of bounded linear operators is denoted by $\mathcal{L}(E ; F)$, and $\mathcal{L}(E)$ if $E=F$; the space of compact operators is $\mathcal{K}(E ; F)$, and $\mathcal{K}(E)$ if $E=F$. Frequently, we shall endow $\mathcal{L}(E ; F)$ with the strong operator topology (sot). To indicate this we use terms like "sot-closed", "sot-compact" or speak of "strongly closed" or "strongly compact" sets etc. A similar convention applies when the weak operator topology (wot) is considered. Whereas for a set $A \subseteq E$ the set $\operatorname{cl}_{\sigma}(A)$ is the closure of $A$ in the weak $\left(=\sigma\left(E ; E^{\prime}\right)\right)$ topology on $E$, the sot-closure and the wot-closure of a set $M \subseteq \mathcal{L}(E ; F)$ are denoted by

$$
\operatorname{cl}_{\mathrm{s}}(M) \text { and } \operatorname{cl}_{\mathrm{w}}(M),
$$

respectively. We shall frequently use the following auxiliary result, see EN00, Corollary A.5].

Lemma 1.1. Let $E$ be a Banach space. Then a bounded subset $M \subseteq \mathcal{L}(E)$ is relatively strongly (weakly) compact if and only if the orbit

$$
M x:=\{T x \mid T \in M\}
$$

is relatively (weakly) compact for all $x$ from a dense subset of $E$.

The set $\mathcal{L}(E)$ is a semigroup with respect to operator multiplication. Operator multiplication is sot- and wot-separately continuous, and it is sot-simultaneously continuous on norm-bounded sets.

For the definition of a semigroup as well as for some elementary definitions and results from algebraic semigroup theory, see Appendix C

We shall freely use standard results and notation from the theory of Banach lattices, with Sch74] and MN91] being our main references. If $E$ is a Banach lattice the set $E_{+}:=\{x \in E \mid x \geq 0\}$ is its cone of positive elements. In some proofs we confine tacitly to real Banach lattices, but there should be no difficulty to extend the arguments to the complex case.

\section{Representations of Abelian Semigroups}

Throughout the article, $S$ is an Abelian semigroup (written additively) containing a neutral element 0 .

Observe that for each $s \in S$ the set

$$
s+S:=\{s+r \mid r \in S\} \subseteq S
$$


is a subsemigroup of $S$. We turn $S$ into a directed set by letting

$$
s \leq t \quad \stackrel{\text { def. }}{\Longleftrightarrow} t \in s+S \Longleftrightarrow t+S \subseteq s+S .
$$

For limits of nets $\left(x_{s}\right)_{s \in S}$ with respect to this direction, the notation $\lim _{s \in S} x_{s}$ is used. Note that $0 \leq s$ for all $s \in S$.

Example. Observe that in the cases $S=\mathbb{Z}_{+}$and $S=\mathbb{R}_{+}$the so-defined direction and the associated notion of limit coincides with the usual one.

A representation of $S$ on a Banach space $E$ is any mapping $T: S \rightarrow \mathcal{L}(E)$ satisfying

$$
T(0)=\mathrm{I} \quad \text { and } \quad T(s+t)=T(s) T(t) \quad(t, s \in S) .
$$

In place of $T(s)$ we also use index notation $T_{s}$, and often call $T=\left(T_{s}\right)_{s \in S}$ an operator semigroup (over $S$ on $E$ ). The fixed space of the representation $T$ is

$$
\operatorname{fix}(T):=\bigcap_{s \in S} \operatorname{ker}\left(T_{s}-\mathrm{I}\right)=\left\{x \in E \mid T_{s} x=x \text { for all } s \in S\right\}
$$

An operator semigroup $\left(T_{s}\right)_{s \in S}$ is bounded if

$$
M_{T}:=\sup _{s \in S}\left\|T_{s}\right\|<\infty .
$$

Boundedness has the following useful consequence.

Lemma 2.1. Let $T=\left(T_{s}\right)_{s \in S}$ be a bounded operator semigroup on the Banach space $E$. Then for each vector $x \in E$ the following assertions are equivalent:

(i) $0 \in \overline{\left\{T_{s} x \mid s \in S\right\}}$.

(ii) $\lim _{s \in S} T_{s} x=0$.

Proof. Suppose that (i) holds and $\varepsilon>0$. Then there is $s \in S$ such that $\left\|T_{s} x\right\| \leq \varepsilon$. But then

$$
\left\|T_{t} x\right\| \leq \varepsilon M_{T} \quad \text { for all } t \in s+S .
$$

It follows that $\lim _{s \in S} T_{s} x=0$, i.e., (ii). The converse is trivial.

Given an operator semigroup $T=\left(T_{s}\right)_{s \in S}$ on a Banach space $E$, a subset $A$ of $E$ is called $T$-invariant if $T_{s}(A) \subseteq A$ for all $s \in S$. A closed, $T$-invariant subspace $F$ of $E$ gives rise to a subrepresentation by restricting the operators $T_{s}$ to $F$. Such a subrepresentation is called finite-dimensional ( $d$-dimensional) $(d \in \mathbb{N})$ if $F$ is finite-dimensional ( $d$-dimensional).

A one-dimensional subrepresentation is given by a scalar representation $\lambda: S \rightarrow$ $\mathbb{K}$ and a non-zero vector $u \in E$ such that

$$
T_{s} u=\lambda_{s} u \quad \text { for all } s \in S .
$$

The corresponding mapping $\lambda$ is then called an eigenvalue of $T$, and $u$ is called a corresponding eigenvector. Obviously,

$$
\lambda \text { is constant } \Leftrightarrow \quad \lambda=\mathbf{1} \quad \Leftrightarrow \quad u \in \operatorname{fix}(T) .
$$

An eigenvalue $\lambda=\left(\lambda_{s}\right)_{s \in S}$ is called unimodular if $\left|\lambda_{s}\right|=1$ for each $s \in S$. (So the constant eigenvalue $\mathbf{1}$ is unimodular.) An unimodular eigenvalue $\lambda$ of $T$ is called a torsion eigenvalue if there is $m \in \mathbb{N}$ such that $\lambda_{s}^{m}=1$ for all $s \in S$.

If $E$ is a Banach lattice, a semigroup $\left(T_{s}\right)_{s \in S}$ on $E$ is called positive if the positive cone $E_{+}$is $T$-invariant, i.e., if each operator $T_{s}$ is positive. And a positive 
semigroup is called irreducible or said to act irreducibly on $E$ if $\{0\}$ and $E$ are the only $T$-invariant closed ideals of $E$. (Recall that a subspace $J$ of $E$ is an ideal if it satisfies: $x \in E, y \in J,|x| \leq|y| \quad \Rightarrow \quad x \in J$.)

The Semigroup at Infinity. Given an operator semigroup $T=\left(T_{s}\right)_{s \in S}$ we write

$$
T_{S}:=\left\{T_{s} \mid s \in S\right\} \subseteq \mathcal{L}(E)
$$

for its range, which is a subsemigroup of $\mathcal{L}(E)$. And we abbreviate

$$
\mathcal{T}:=\operatorname{cl}_{\mathrm{s}}\left\{T_{s} \mid s \in S\right\} \quad \text { and } \quad \mathcal{T}_{s}:=\operatorname{cl}_{\mathrm{s}}\left\{T_{t} \mid t \geq s\right\} \quad(s \in S)
$$

and call

$$
\mathcal{T}_{\infty}:=\bigcap_{s \in S} \mathcal{T}_{s}=\bigcap_{s \in S} \operatorname{cl}_{s}\left\{T_{t} \mid t \geq s\right\}
$$

the associated semigroup at infinity. In effect, $\mathcal{T}_{\infty}$ is the set of sot-cluster points of the net $\left(T_{s}\right)_{s \in S}$.

Note that $\mathcal{T}_{\infty}$ is multiplicative and even satifies

$$
\mathcal{T} \cdot \mathcal{T}_{\infty} \subseteq \mathcal{T}_{\infty}
$$

But it may be empty (in which case it is, according to our definition in Appendix C] not a semigroun].)

The JdLG-Splitting Theory. One of the principal methods to prove strong convergence of a bounded semigroup is to employ the splitting theory of Jacobs, de Leeuw and Glicksberg as detailed, e.g., in EFHN15, Chapter 16]. Usually, this theory is applied to the semigroup $\mathcal{T}$ or to its wot-counterpart $\operatorname{cl}_{\mathrm{w}}\left\{T_{s} \mid s \in S\right\}$. In contrast, we shall apply it to $\mathcal{T}_{\infty}$. If $\mathcal{T}_{\infty}$ is a strongly compact semigroup, the JdLG-theory tells that it contains a unique minimal idempotent, which we denote by $P_{\infty}$. (Minimality means that $P_{\infty} \cdot \mathcal{T}_{\infty}$ is a minimal ideal in $\mathcal{T}_{\infty}$.) The range of $P_{\infty}$ is denoted here by

$$
E_{\infty}:=\operatorname{ran}\left(P_{\infty}\right) .
$$

Observe that $Q T_{s}=T_{s} Q$ for each $s \in S$ and each $Q \in \mathcal{T}_{\infty}$. In particular, $E_{\infty}$ is $\mathcal{T}$-invariant.

Theorem 2.2. Let $T=\left(T_{s}\right)_{s \in S}$ be a bounded operator semigroup on the Banach space $E$ such that the associated semigroup at infinity, $\mathcal{T}_{\infty}$, is strongly compact and non-empty. Then the following additional assertions hold:

a) $\mathcal{T} P_{\infty}=\mathcal{T}_{\infty} P_{\infty}$

b) $T$ is relatively strongly compact on $E_{\infty}$, i.e.,

$$
\mathcal{G}:=\operatorname{cl}_{\mathrm{s}}\left\{\left.T_{s}\right|_{E_{\infty}} \mid s \in S\right\} \subseteq \mathcal{L}\left(E_{\infty}\right)
$$

is a strongly compact group of invertible operators on $E_{\infty}$. Moreover,

$$
\mathcal{G}=\left.\mathcal{T}\right|_{E_{\infty}}:=\left\{\left.Q\right|_{E_{\infty}} \mid Q \in \mathcal{T}\right\} .
$$

c) For each $x \in E$ the following statements are equivalent:

(i) $P_{\infty} x=0$

(ii) $0 \in \operatorname{cl}_{\sigma}\left\{T_{s} x \mid s \in S\right\}$.

(iii) $\lim _{s \in S} T_{s} x=0$.

(iv) $R x=0$ for some $/$ all $R \in \mathcal{T}_{\infty}$.

\footnotetext{
${ }^{1}$ We apologize for this little abuse of terminology.
} 
d) If $\left(\lambda_{s}\right)_{s \in S}$ is a unimodular eigenvalue of $T$ with eigenvector $0 \neq x \in E$, then $x \in E_{\infty}$ and there is a unique eigenvalue $\mu=\left(\mu_{Q}\right)_{Q \in \mathcal{G}}$ of $\mathcal{G}$ such that $\lambda_{s}=\mu_{T_{s}}$ for all $s \in S$.

e) If $\mu=\left(\mu_{Q}\right)_{Q \in \mathcal{G}}$ is an eigenvalue of $\mathcal{G}$ on $E_{\infty}$, then $\lambda_{s}:=\mu_{T_{s}}(s \in S)$ is an unimodular eigenvalue of $T$.

(We suppose $\mathbb{K}=\mathbb{C}$ for assertions $\mathrm{d}$ ) and $\mathrm{e}$ ).)

Proof. a) Since $\mathcal{T} \mathcal{T}_{\infty} \subseteq \mathcal{T}_{\infty} \mathcal{T}$, we have $\mathcal{T} P_{\infty}=\mathcal{T} P_{\infty} P_{\infty} \subseteq \mathcal{T}_{\infty} P_{\infty} \subseteq \mathcal{T} P_{\infty}$.

b) By a) we have $\left.\mathcal{T}\right|_{E_{\infty}}=\left.\mathcal{T}_{\infty}\right|_{E_{\infty}}$, and the latter is a strongly compact group of invertible operators on $E_{\infty}$ by the JdLG-theory. Since restriction is a sot-continuous operator from $\mathcal{L}(E)$ to $\mathcal{L}\left(E_{\infty} ; E\right),\left.\mathcal{T}\right|_{E_{\infty}} \subseteq \mathcal{G}$. The converse inclusion follows from $\mathcal{G} P_{\infty} \subseteq \mathcal{T}$, which is true because $P_{\infty} \in \mathcal{T}$.

c) If $R x=0$ for all $R \in \mathcal{T}_{\infty}$, then clearly (i) holds, and (i) implies that $R x=0$ for some $R \in \mathcal{T}_{\infty}$. On the other hand, this latter statement obviously implies $0 \in \overline{\left\{T_{s} x \mid s \in S\right\}}$, which is equivalent to $\lim _{s \in S} T_{s} x=0$, i.e., (iii).

If, in turn, (iii) holds and $\varepsilon>0$ is fixed, then there is $s \in S$ such that $\left\{T_{t} x \mid t \geq\right.$ $s\} \subseteq \mathrm{B}[0, \varepsilon]$. Hence, also $\mathcal{T}_{\infty} x \subseteq \mathrm{B}[0, \varepsilon]$. As $\varepsilon>0$ was arbitrary, $\mathcal{T}_{\infty} x=\{0\}$, i.e., $R x=0$ for all $R \in \mathcal{T}_{\infty}$.

Finally, (iii) obviously implies (ii). Conversely, starting from (ii) we apply $P_{\infty}$ to obtain

$$
0 \in \mathrm{cl}_{\sigma}\left\{T_{s} P_{\infty} x \mid s \in S\right\} .
$$

However, by b) the set $\left\{T_{s} P_{\infty} x \mid s \in S\right\}$ is relatively strongly compact and hence its weak and its strong closures must coincide. This yields

$$
0 \in \overline{\left\{T_{s} P_{\infty} x \mid s \in S\right\}}
$$

which implies $P_{\infty} x=P_{\infty}\left(P_{\infty} x\right)=0$ by what we have already shown.

d) Let $0 \neq x \in E$ be an eigenvector for the unimodular eigenvalue $\left(\lambda_{s}\right)_{s \in S}$ of $T$. Define $y:=x-P_{\infty} x$. Then $P_{\infty} y=0$ and hence $T_{s} y \rightarrow 0$. On the other hand, since $P_{\infty}$ commutes with every $T_{s}, T_{s} y=\lambda_{s} y$ for all $s \in S$. As $\left|\lambda_{s}\right|=1$, it follows that $y=0$ and hence $x \in E_{\infty}$. The remaining statement now follows easily since $\mathbb{C} x$ is $T$-invariant and $\left.T_{S}\right|_{E_{\infty}}$ is dense in $\mathcal{G}$.

e) is obvious.

As a corollary we obtain the following characterization of the strong convergence of a semigroup.

Corollary 2.3. For a bounded operator semigroup $T=\left(T_{s}\right)_{s \in S}$ on a Banach space $E$ the following assertions are equivalent:

(i) $T$ is strongly convergent;

(ii) $\mathcal{T}_{\infty}$ is a singleton;

(iii) $\mathcal{T}_{\infty}$ is non-empty and strongly compact and acts as the identity on $E_{\infty}$;

(iv) $\mathcal{T}_{\infty}$ is non-empty and strongly compact and $T$ acts as the identity on $E_{\infty}$.

In this case: $\lim _{s \in S} T_{s}=P_{\infty}$.

Proof. (i) $\Rightarrow$ (ii): If $T$ is strongly convergent with $P:=\lim _{s \in S} T_{s}$ being its limit, then $\mathcal{T}_{\infty}=\{P\}$ is a singleton.

(ii) $\Rightarrow$ (iii): If $\mathcal{T}_{\infty}=\{P\}$ is a singleton, then it is clearly non-empty and strongly compact. It follows that $P=P_{\infty}$, and hence $\mathcal{T}_{\infty}$ acts as the identity on $E_{\infty}$. 
(iii) $\Rightarrow$ (iv): Suppose that $\mathcal{T}_{\infty}$ is non-empty and strongly compact and acts as the identity on $E_{\infty}$. Let $Q \in \mathcal{T}_{\infty}$. Then, by the equivalence (i) $\Leftrightarrow$ (iv) in Theorem 2.2. c), $Q\left(\mathrm{I}-P_{\infty}\right)=0$ and hence $Q=Q P_{\infty}=P_{\infty}$. So it follows that $\mathcal{T}_{\infty}=\left\{P_{\infty}\right\}$. Moreover, since $T_{s} \mathcal{T}_{\infty} \subseteq \mathcal{T}_{\infty}$ for each $s \in S$, we obtain $T_{s} P_{\infty}=P_{\infty}$ and hence $T_{s}=\mathrm{I}$ on $E_{\infty}$ for all $s \in S$.

(iv) $\Rightarrow$ (i): Suppose that (iv) holds. Then

$$
\lim _{s \in S} T_{s}=\lim _{s \in S}\left(T_{s} P_{\infty}+T_{s}\left(\mathrm{I}-P_{\infty}\right)\right)=P_{\infty}+\lim _{s \in S} T_{s}\left(\mathrm{I}-P_{\infty}\right)=P_{\infty}
$$

strongly, by the equivalence (i) $\Leftrightarrow$ (iii) of Theorem $2.2 \mathrm{c}$ ).

Theorem 2.2 and its corollary yield the following strategy to prove strong convergence of an operator semigroup:

1) Show that $\mathcal{T}_{\infty}$ is non-empty and strongly compact.

2) Show that $\mathcal{T}_{\infty}$ (or, equivalently, $T$ ) acts as the identity on $E_{\infty}:=\operatorname{ran}\left(P_{\infty}\right)$. (For this one may employ the additional information that $\mathcal{T}$ acts on $E_{\infty}$ as a compact group.)

Remark 2.4. Suppose that $T=\left(T_{s}\right)_{s \in S}$ is a strongly relatively compact operator semigroup on $E$ with minimal idempotent $P \in \mathcal{T}$. Then, of course, $\mathcal{T}_{\infty}$ is nonempty and strongly compact, and hence a closed ideal of $\mathcal{T}$. It follows from the minimality of $P$ in $\mathcal{T}$ and $P_{\infty}$ in $\mathcal{T}_{\infty}$ that

$$
P \mathcal{T} \subseteq P_{\infty} \mathcal{T} \subseteq P_{\infty} \mathcal{T}_{\infty} \subseteq P \mathcal{T}_{\infty} \subseteq P \mathcal{T}
$$

Hence $P \mathcal{T}=P_{\infty} \mathcal{T}_{\infty}$, which implies that $P=P_{\infty}$. So, in the case that $T$ is relatively strongly compact, passing to the semigroup at infinity yields the same JdLG-decomposition of $E$ as working with $\mathcal{T}$.

We end this section with a technical, but useful characterization of the property that $\mathcal{T}_{\infty}$ is non-empty and compact.

Proposition 2.5. For a bounded operator semigroup $T=\left(T_{s}\right)_{s \in S}$ on a Banach space $E$ the following assertions are equivalent:

(i) $\mathcal{T}_{\infty}$ is non-empty and strongly compact.

(ii) Every subnet of $\left(T_{s}\right)_{s \in S}$ has a strongly convergent subnet.

(iii) Every universal subnet of $\left(T_{s}\right)_{s \in S}$ is strongly convergent.

(iv) For each $x \in E$ every subnet of $\left(T_{s} x\right)_{s \in S}$ has a convergent subnet.

(v) For each $x \in E$ every universal subnet of $\left(T_{s} x\right)_{s \in S}$ converges.

If $S$ contains a cofinal sequence, then the above assertions are also equivalent to:

(vi) For every $x \in E$ and every cofinal sequence $\left(s_{n}\right)_{n \in \mathbb{N}} \subseteq S$, the sequence $\left(S_{s_{n}} x\right)_{n \in \mathbb{N}}$ has a convergent subsequence.

Proof. (i) $\Rightarrow$ (iv): Suppose that (i) holds and let $x \in E$. The net $\left(T_{s}\left(\mathrm{I}-P_{\infty}\right) x\right)_{s \in S}$ converges to 0 according to Theorem $2.2 \mathrm{c})$. On the other hand, the net $\left(T_{s} P_{\infty} x\right)_{s \in S}$ is contained in the compact set $\mathcal{T}_{\infty} P_{\infty} x$ due to Theorem $2.2 \mathrm{a}$ ), so each of its subnets has a convergent subnet. This shows (iv).

(iv) $\Rightarrow(\mathrm{v})$ : This follows since a universal net with a convergent subnet must converge. 
(v) $\Rightarrow$ (iii): Let $\left(T_{s_{\alpha}}\right)_{\alpha \in I}$ be a universal subnet of $\left(T_{s}\right)_{s \in S}$. Then for each $x \in E$, the net $\left(T_{s_{\alpha}} x\right)_{\alpha \in I}$ is universal and hence, by (v), convergent. Thus, $\left(T_{s_{\alpha}}\right)_{\alpha \in I}$ is strongly convergent.

(iii) $\Rightarrow$ (ii) $\Rightarrow$ (i) and (iv) $\Rightarrow$ (vi) all follow from Theorem B.3.

(vi) $\Rightarrow$ (i): Suppose that $S$ admits a cofinal sequence. Then by Theorem B.3 for each $x \in E$ the set $C_{x}:=\bigcap_{t \in S} \operatorname{cl}\left\{T_{s} x \mid s \geq t\right\}$ is non-empty and compact. Since $\mathcal{T}_{\infty} x \subseteq C_{x}$, it follows that $\mathcal{T}_{\infty}$ is strongly compact. In order to see that $\mathcal{T}_{\infty}$ is not empty, fix a cofinal sequence $\left(s_{n}\right)_{n}$. By (vi) and since $E$ is metrizable, it follows that for each $x \in E$ the set $\left\{T_{s_{n}} x \mid n \in \mathbb{N}\right\}$ is relatively compact. Hence, $\left\{T_{s_{n}} \mid n \in \mathbb{N}\right\}$ is relatively strongly compact. It follows that the sequence $\left(T_{s_{n}}\right)_{n}$ has a cluster point, which is a member of $\mathcal{T}_{\infty}$ since $\left(s_{n}\right)_{n}$ is cofinal.

Remarks 2.6. 1) Assertion (vi) in Proposition 2.5 is called strong asymptotic compactness in [EKRW01, p. 2636].

2) Proposition 2.5 has an interesting consequence in the "classical" cases where $S=\mathbb{N}_{0}$ or $S=\mathbb{R}_{+}$and $T$ is strongly continuous (cf. the Introduction). Namely, in these cases one can actually dispense with the semigroup at infinity, because $\mathcal{T}_{\infty}$ is strongly compact and non-empty if and only if $\mathcal{T}$ is strongly compact.

In the next section we shall present another situation in which $\mathcal{T}_{\infty}$ is non-empty and strongly compact.

\section{The Abstract Main Result}

Suppose that $E$ and $F$ are Banach spaces such that $F$ is densely embedded in E:

$$
F \stackrel{d}{\hookrightarrow} E .
$$

Reference to this embedding is usually suppressed and $F$ is simply regarded as a subspace of $E$. We take the freedom to consider an operator on $E$ also as an operator from $F$ to $E$. (This amounts to view $\mathcal{L}(E) \subseteq \mathcal{L}(F ; E)$ via the restriction mapping.)

A semigroup $\left(T_{s}\right)_{s \in S}$ on $E$ is called $F$-to- $E$ quasi-compact, or quasi-compact relatively to $F$, if for each $\varepsilon>0$ there is $s \in S$ and a compact operator $K: F \rightarrow E$ such that

$$
\left\|T_{s}-K\right\|_{\mathcal{L}(F ; E)}<\varepsilon \text {. }
$$

Note that we do not require that $K$ can be extended to a bounded operator on $E$. In effect, the condition of being $F$-to- $E$ quasi-compact can be expressed as

$$
\operatorname{dist}\left(\left\{T_{s} \mid s \in S\right\}, \mathcal{K}(F ; E)\right)=0,
$$

where "dist" refers to the distance induced by the norm on $\mathcal{L}(F ; E)$.

Theorem 3.1. Let $E$ and $F$ be Banach spaces, with $F$ being densely embedded into $E$, and let $\left(T_{s}\right)_{s \in S}$ be a bounded operator semigroup on $E$ which restricts to a bounded operator semigroup on $F$ and is $F$-to-E quasi-compact. Then the following assertions hold:

a) $\mathcal{T}_{\infty}$ is a strongly compact and non-empty.

b) Each element of $\mathcal{T}_{\infty}$ is compact as an operator from $F$ to $E$.

c) $\mathcal{T}$ acts on $E_{\infty}$ as a sot-compact group of invertible operators. 
d) For $x \in E$ the following assertions are equivalent:

(i) $\lim _{s \in S} T_{s} x=0$;

(ii) $x \in \operatorname{ker} P_{\infty}$;

(iii) $0 \in \operatorname{cl}_{\sigma}\left\{T_{s} x \mid s \in S\right\}$.

Proof. a) and b) By passing to an equivalent norm on $F$ we may suppose that each $T_{s}, s \in S$, is a contraction on $F$. Let $\mathrm{B}_{F}$ and $\mathrm{B}_{E}$ denote the closed unit balls of $E$ and $F$, respectively.

Let $\varepsilon>0$ and choose $s \in S$ and $K \in \mathcal{K}(F ; E)$ such that $\left\|T_{s}-K\right\|_{\mathcal{L}(F ; E)} \leq \varepsilon$. Then

$$
T_{t+s}\left(\mathrm{~B}_{F}\right)=T_{s} T_{t}\left(\mathrm{~B}_{F}\right) \subseteq T_{s}\left(\mathrm{~B}_{F}\right) \subseteq K\left(\mathrm{~B}_{F}\right)+\varepsilon \mathrm{B}_{E}
$$

for each $t \in S$, and therefore

$$
\mathcal{T}_{s}\left(\mathrm{~B}_{F}\right) \subseteq \overline{K\left(\mathrm{~B}_{F}\right)}+\varepsilon \mathrm{B}_{E} .
$$

Now, let $\left(T_{s_{\alpha}}\right)_{\alpha}$ be any universal subnet of $\left(T_{s}\right)_{s \in S}$ (Lemma B.1) and let $x \in \mathrm{B}_{F}$. Then the net $\left(T_{s_{\alpha}} x\right)_{\alpha}$ is a universal net in $E$. Moreover, (3.1) shows that for each $\varepsilon>0$ this net has a tail contained in the $\varepsilon$-neighborhood of some compact set. Hence, by Lemma B.2 it is a Cauchy net and thus convergent in $E$. Since $F$ is dense in $E$ and $T$ is bounded, $\left(T_{s_{\alpha}} x\right)_{\alpha}$ converges for every $x \in E$. In other words, $\left(T_{s_{\alpha}}\right)_{\alpha}$ is strongly convergent. As its limit must be a member of $\mathcal{T}_{\infty}$, it follows that $\mathcal{T}_{\infty} \neq \emptyset$.

It also follows from (3.1) that $\mathcal{T}_{\infty}\left(\mathrm{B}_{F}\right) \subseteq \overline{K\left(\mathrm{~B}_{F}\right)}+\varepsilon \mathrm{B}_{E}$. As $\overline{K\left(\mathrm{~B}_{F}\right)}$ is compact, it admits a finite $\varepsilon$-mesh. Hence, $\mathcal{T}_{\infty}\left(\mathrm{B}_{F}\right)$ admits a finite $2 \varepsilon$-mesh. Since this works for each $\varepsilon>0, \mathcal{T}_{\infty}\left(\mathrm{B}_{F}\right)$ is relatively compact in $E$.

In particular, it follows that $\mathcal{T}_{\infty} \subseteq \mathcal{K}(F ; E)$ and that for each $x \in F$ the orbit $\mathcal{T}_{\infty} x$ is relatively compact in $E$. Since $T$ is bounded on $E$ and $F$ is dense in $E$, $\mathcal{T}_{\infty}$ is relatively strongly compact (Lemma 1.1). But $\mathcal{T}_{\infty}$ is strongly closed, so it is strongly compact as claimed.

c) and d) follow from a) by Theorem 2.2 .

Remark 3.2. Theorem 3.1 seems to be new even for $C_{0}$-semigroups. In that case, by Remark 2.6. b), it follows a posteriori that the $C_{0}$-semigroup is relatively compact.

In the next sections we shall see that our set-up from above has a quite natural instantiation in the context of semigroups of positive operators on Banach lattices with a quasi-interior point.

\section{Positive Semigroups and AM-Compactness}

From now on, we consider positive semigroups $T=\left(T_{s}\right)_{s \in S}$ on Banach lattices $E$. The role of $F$ in our abstract setting from above will be taken by the principal ideal

$$
E_{y}:=\{x \in E \mid \text { there is } c \geq 0 \text { such that }|x| \leq c y\}
$$

for some $y \in E_{+}$, endowed with the natural AM-norm

$$
\|x\|_{y}:=\inf \{c \geq 0|| x \mid \leq c y\} .
$$

Since we need that $F=E_{y}$ is dense in $E$, we have to require that $y$ is a quasiinterior point in $E$.

As we further need $E_{y}$-to- $E$ quasi-compactness, it is natural to ask which operators on $E$ restrict to compact operators from $E_{y}$ to $E$. It turns out that these 
are precisely the $A M$-compact operators, i.e., those that map order intervals of $E$ to relatively compact subsets of $E$, see Lemma A.3

There are a couple of useful theorems that help to identify AM-compact operators. For example, operators between $\mathrm{L}^{p}$-spaces induced by positive integral kernel functions and positive operators that "factor through $\mathrm{L}^{\infty}$-spaces" are AM-compact. (Proofs of these well-known facts are presented in Appendix A, see Theorems A.3 and A.4.)

The Range of a Positive Projection. When we apply Theorem 3.1 to a semigroup of positive operators, the resulting projection $P_{\infty}$ will be positive, too. The following is a useful information about its range.

Lemma and Definition 4.1. Let $E$ be a Banach lattice and let $P$ be a positive projection on E. Define

$$
\|x\|_{P}:=\|P|x|\| \quad(x \in \operatorname{ran}(P)) .
$$

Then the following assertions hold:

a) $\|\cdot\|_{P}$ is an equivalent norm on $\operatorname{ran}(P)$.

b) The space $\operatorname{ran}(P)$ is a Banach lattice with respect to the order induced by $E$ and the norm $\|\cdot\|_{P}$. Its modulus is given by

$$
|x|_{P}:=P|x| \quad(x \in \operatorname{ran}(P)) .
$$

The Banach lattice $\operatorname{ran}(P)$ endowed with the norm $\|\cdot\|_{P}$ as in a) and b) is denoted by $[\operatorname{ran}(P)]$.

c) If $y \in E_{+}$then $P\left(E_{y}\right) \subseteq[\operatorname{ran}(P)]_{P y}$. In particular, if $y \in E_{+}$is a quasiinterior point of $E$ then $P y$ is a quasi-interior point of $[\operatorname{ran}(P)]$.

Proof. This is essentially [Sch74, Proposition III.11.5].

In order to obtain further insight into the relation of the closed ideals in $[\operatorname{ran}(P)]$ and in $E$, we define for any Banach lattice $E$ the mapping

$$
\Phi(J):=\operatorname{cl}\left\{x \in E|| x \mid \leq y \text { for some } y \in J_{+}\right\} \quad(J \subseteq E) .
$$

If $J_{+}=J \cap E_{+}$is a cone, then $\Phi(J)=\Phi\left(J_{+}\right)$is the smallest closed ideal in $E$ containing $J_{+}$.

Theorem 4.2. Let $E$ be a Banach lattice and $P$ a positive projection on $E$, and let $\Phi$ be defined as above. Then the following assertions hold:

a) If $I$ is a closed $P$-invariant ideal in $E$ then $P(I)=I \cap \operatorname{ran}(P)$ is a closed ideal in $[\operatorname{ran}(P)]$.

b) If $J$ is a closed ideal in $[\operatorname{ran}(P)]$ then $\Phi(J)$ is $P$-invariant and the smallest closed ideal in E containing J. Moreover,

$$
J=P(\Phi(J))=\Phi(J) \cap \operatorname{ran}(P) .
$$

Proof. a) Let $I \subseteq E$ be a closed $P$-invariant ideal and let $J:=I \cap \operatorname{ran}(P)$. Then $J$ is a closed subspace of $\operatorname{ran}(P)$. And if $x \in \operatorname{ran}(P)$ and $y \in J$ with $|x|_{P} \leq|y|_{P}$, it follows that

$$
|x|=|P x| \leq P|x|=|x|_{P} \leq|y|_{P}=P|y| \in I
$$


by $P$-invariance. Hence, $x \in J$ and therefore $J$ is an ideal in $[\operatorname{ran}(P)]$. Moreover, again by $P$-invariance,

$$
J=P J=P(I \cap \operatorname{ran}(P)) \subseteq P(I) \subseteq I \cap \operatorname{ran}(P),
$$

and hence $I \cap \operatorname{ran}(P)=J=P(I)$.

b) Let $J$ be any closed ideal of $[\operatorname{ran}(P)]$. Then $\Phi(J)$ is the smallest closed ideal in $E$ containing $J$. (In fact, if $x \in J$ then $|x|=|P x| \leq P|x|=|x|_{P} \in J_{+}$, and hence $J \subseteq \Phi(J)$.) It is also $P$-invariant, for if $|x| \leq y \in J_{+}$then $|P x| \leq P|x| \leq P y=y$. This also shows that $P(\Phi(J)) \subseteq J$, and since $J \subseteq \Phi(J)$, it follows that $P(\Phi(J))=$ $J$.

The Main Result for Positive Semigroups. We are now prepared for our second main theorem.

Theorem 4.3. Let $T=\left(T_{s}\right)_{s \in S}$ be a bounded and positive operator semigroup on a Banach lattice $E$ with a quasi-interior point $y \in E_{+}$. Suppose, in addition, that $T$ is $E_{y}$-to-E quasi-compact and restricts to a bounded semigroup on $E_{y}$. Then the following assertions hold:

a) $\mathcal{T}_{\infty}$ is strongly compact and non-empty and consists of AM-compact operators.

b) $\left[\operatorname{ran}\left(P_{\infty}\right)\right]$ is an atomic Banach lattice with order continuous norm and quasiinterior point $P_{\infty} y$.

c) The semigroup $\mathcal{T}=\operatorname{cl}_{\mathrm{s}}\left\{T_{s} \mid s \in S\right\}$ acts on $\left[\operatorname{ran}\left(P_{\infty}\right)\right]$ as a compact topological group of positive, invertible operators.

d) If $\left(T_{s}\right)_{s \in S}$ acts irreducibly on $E$, then $\mathcal{T}$ acts irreducibly on $\left[\operatorname{ran}\left(P_{\infty}\right)\right]$.

Proof. a) follows from Theorem 3.1 and Lemma A.1.

b) Each order interval $J$ of $E_{\infty}=\left[\operatorname{ran}\left(P_{\infty}\right)\right]$ is of the form $J=J^{\prime} \cap E_{\infty}$, where $J^{\prime}$ is an order interval of $E$. Since $P_{\infty}$ is AM-compact but restricts to the identity on $E_{\infty}$, it follows that $J=P_{\infty}(J) \subseteq P_{\infty}\left(J^{\prime}\right)$ is relatively compact. By Wnu99, Theorem 6.1], this implies that $E_{\infty}$ as a Banach lattice is atomic and has order continuous norm.

c) This follows again from Theorem 3.1

d) Suppose that $J \neq\{0\}$ is a closed $\mathcal{T}$-invariant ideal in $\left[\operatorname{ran}\left(P_{\infty}\right)\right]$. Then the set

$$
\left\{x \in E|| x \mid \leq y \text { for some } y \in J_{+}\right\}
$$

is $T$-invariant, and hence $\Phi(J)$ is a closed $T$-invariant ideal in $E$ containing $J$. By irreducibility, $\Phi(J)=E$, and hence $\operatorname{ran}\left(P_{\infty}\right)=P(E)=P(\Phi(J))=J$ by Theorem 4.2 .

Remarks 4.4. 1) The assumption that $T$ restricts to a bounded semigroup on $E_{y}$ is for instance satisfied if $y$ is a sub-fixed point of $T$, i.e., if $T_{t} y \leq y$ for all $t \in S$.

2) Certainly, if $T_{s}$ is AM-compact for some $s \in S$ then $T$ is $E_{y}$-to- $E$ quasicompact.

We conclude this section with the following result, essentially proved by Gerlach and Glück in GG17b, Lemma 3.12]. It shows that in certain situations it suffices to require merely that $T_{s}$ dominates a non-trivial AM-compact operator for some $s \in S$. 
Lemma 4.5. Let $T=\left(T_{s}\right)_{s \in S}$ be a bounded, positive and irreducible semigroup on a Banach lattice $E$ with order continuous norm and a quasi-interior sub-fixed point $y$ of $T$. Suppose that there are $s \in S$ and an AM-compact operator $K \neq 0$ on $E$ with $0 \leq K \leq T_{s}$. Then $T$ is $E_{y}$-to-E quasi-compact and restricts to a bounded semigroup on $E_{y}$. In particular, Theorem 4.3 is applicable.

\section{Compact Groups of Positive Operators on Atomic Banach Lattices}

In view of our general strategy, Theorem 4.3 suggests to look for criteria implying that a positive group representation on an atomic Banach lattice with ordercontinuous norm is trivial. To this end, we first summarize some known results about atomic Banach lattices.

Atomic Banach Lattices. Recall that an atom in a Banach lattice is any element $0 \neq a \in E$ such that its generated principal ideal, $E_{a}$, is one-dimensional: $E_{a}=\mathbb{K} \cdot a$. We denote by

$$
A=A_{E}:=\left\{a \in E_{+} \mid a \text { atom, }\|a\|=1\right\}
$$

the set of positive atoms of norm one. For distinct $a, b \in A$ one has

$$
|a-b|=|a+b|=a+b \geq a
$$

and hence $\|a-b\| \geq\|a\|=1$. This shows that $A$ is a discrete set with respect to the norm topology.

A Banach lattice $E$ is called atomic, if $E$ is the smallest band in $E$ that contains all atoms. In other words,

$$
A^{d}:=\{x \in E|| x \mid \wedge a=0 \text { for all } a \in A\}=\{0\} .
$$

For each $a \in A$ the one-dimensional subspace $E_{a}=\mathbb{K} a$ is a projection band, with corresponding band projection $P_{A}$ given by

$$
P_{a} x:=\sup [0, x] \cap \mathbb{R} a=\sup \left\{t \in \mathbb{R}_{+} \mid t a \leq x\right\} \cdot a \quad\left(x \in E_{+}\right) .
$$

(See, e.g. [LZ71, Thm. 26.4] and cf. [MN91, Prop. 1.2.11].) The next result is a consequence of [Sch74, p.143, Ex. 7] and [MN91, Thm. 1.2.10]. For the convenience of the reader, we give a proof.

Theorem 5.1. Let $E$ be a Banach lattice and let $A$ be its set of positive normalized atoms. Then for each finite subset $F \subseteq A$ the space

$$
\operatorname{span}(F)=\bigoplus_{a \in F} \mathbb{K} a
$$

is a projection band with band projection $P_{F}=\sum_{a \in F} P_{a} . \quad$ Suppose, in addition, that $E$ is atomic. Then

$$
\mathrm{I}_{E}=\sum_{a \in A} P_{a}
$$

as a strongly order convergent series. Each band $B$ in $E$ is generated (as a band) by $A \cap B$. 
Remark 5.2. There are different notions of "order convergence" in the literature, see AS05. We employ the definition found in [MN91, Definition 1.1.9 i)]. For the case of (5.1) this simply means

$$
x=\sup _{F} \sum_{a \in F} P_{a} x \quad \text { for all } x \in E_{+},
$$

where the supremum is taken over all finite subsets of $A$.

Proof of Theorem [5.1. Fix a finite set $F \subseteq A$. If $a, b \in F$ with $a \neq b$, then $a \wedge b=0$ and hence $P_{a} P_{b}=0$. It follows that

$$
P_{F}:=\sum_{a \in F} P_{a}
$$

is a projection (and $F$ is a linearly independent set). Again by the pairwise disjointness of the elements of $F$,

$$
\sum_{a \in F} P_{a} x=\bigvee_{a \in F} P_{a} x \leq x \quad\left(x \in E_{+}\right) .
$$

This shows that $0 \leq P_{F} \leq \mathrm{I}$, and hence $P_{F}$ is a band projection MN91, Lemma 1.2.8]. Since, obviously, $\operatorname{ran}\left(P_{F}\right)=\operatorname{span}(F)$, the first assertion is proved.

In order to prove (5.2) fix $x \in E_{+}$and let $y \in E_{+}$be such that $y \geq P_{F} x$ for all finite $F \subseteq A$. Then $y \geq P_{a} x$ for each $a \in A$ and hence

$$
0 \leq x-(x \wedge y) \leq x-P_{a} x \perp a
$$

If $E$ is atomic, it follows that $x=x \wedge y$, i.e., $x \leq y$. This yields (5.2).

Finally, let $B \subseteq E$ be any band and let $0 \leq x \in B$. Then for each $a \in A$, $P_{a} x \in B$ (since $0 \leq P_{a} x \leq x$ and $B$ is an ideal). Hence, either $P_{a} x=0$ or $a \in B$. It follows from (5.1) that $B$ is generated by $A \cap B$.

With this information at hand we now turn to the representation theory.

A Structure Theorem. Let $G \subseteq \mathcal{L}(E)$ be a group of positive, invertible operators on $E$. (In particular, $G$ consists of lattice homomorphisms.) Then for each $g \in G$ and $a \in A$ the element $g \cdot a \in E$ must be an atom again. In effect

$$
\varphi_{g}(a):=\|g \cdot a\|^{-1}(g \cdot a) \in A \text {. }
$$

It is easy to see that

$$
\varphi: G \rightarrow \operatorname{Sym}(A), \quad g \mapsto \varphi_{g}
$$

is a group homomorphism from $G$ to the group of all bijections on $A$. The corresponding action

$$
G \times A \rightarrow A, \quad(g, a) \mapsto \varphi_{g}(a)
$$

is called the induced action of $G$ on $A$. For each $a \in A$ the orbit mapping

$$
G \rightarrow A, \quad g \mapsto \varphi_{g}(a)
$$

of the induced action is continuous (with respect to the strong operator topology on $G$ ). If, in addition, $G$ is strongly compact, then each orbit

$$
\varphi_{G}(a)=\left\{\varphi_{g}(a) \mid g \in G\right\}
$$

is finite (since $A$ is discrete). We denote by $A / G$ the set of all these orbits. Then $A / G$ is a partition of $A$ into finite subsets. 
Lemma 5.3. In the described situation, suppose that $G$ is compact. Then for $a \in A$ and $g \in G: g \cdot a=a \Leftrightarrow \varphi_{g}(a)=a$. Furthermore: $g=\mathrm{I}_{E} \Leftrightarrow \varphi_{g}=\mathrm{id}_{A}$.

Proof. Fix $a \in A$ and $g \in G$. If $g \cdot a=a$ then $\varphi_{g}(a)=a$, since $\|a\|=1$. Conversely, suppose that $\varphi_{g}(a)=a$. Then $g^{n} \cdot a=\|g \cdot a\|^{n} a$ for all $n \in \mathbb{Z}$. By compactness, $\|g \cdot a\|=1$, and hence $g \cdot a=a$ as claimed.

Suppose that $\varphi_{g}(a)=a$ for all $a \in A$. Then, as we have just seen, $g \cdot a=a$ for all $a \in A$. So $g$ leaves all atoms fixed. Since $g$ acts a lattice isomorphism and hence is order continuous, it follows from Theorem 5.1 that $g=\mathrm{I}_{E}$.

We can now prove a theorem that is reminiscent of the Peter-Weyl structure theorem and its applications to Banach space representations of compact groups.

Theorem 5.4 (Structure Theorem). Let $E \neq\{0\}$ be an atomic Banach lattice and let $A$ be its set of positive normalized atoms. Let $G \subseteq \mathcal{L}(E)$ be a strongly compact group of positive invertible operators on $E$, and let $A / G$ be the set of orbits of elements of $A$ under the induced action of $G$ on $A$. Then the following assertions hold:

a) For each orbit $F \in A / G$ the band $\operatorname{span}(F)$ is $G$-invariant, the corresponding band projection $P_{F}$ is $G$-intertwining, and $G$ acts irreducibly on $\operatorname{span}(F)$.

b) If $B \neq\{0\}$ is a $G$-invariant band in $E$ on which $G$ acts irreducibly, then $B=\operatorname{span}(F)$ for some $F \in A / G$.

c) $I=\sum_{F \in A / G} P_{F}$ as a strongly order-convergent series.

d) In the case $\mathbb{K}=\mathbb{C}$, each eigenvalue of $G$ on $E$ is torsion.

e) If $G$ acts irreducibly on $E$, then $\operatorname{dim}(E)<\infty$ and $G$ has only finitely many eigenvalues.

Proof. a) It is obvious that $\operatorname{span}(F)$ is $G$-invariant and $G$ acts irreducibly on it. Since $G$ consists of lattice automorphisms, also $\operatorname{span}(F)^{d}$ is $G$-invariant, and hence $P_{F}$ is $G$-intertwining.

b) Let $B \neq\{0\}$ be any $G$-invariant band in $E$. Then $B$ is generated (as a band) by $A \cap B$. By $G$-invariance, $A \cap B$ is a union of $G$-orbits (for the induced action), i.e., a union of elements of $A / G$. Hence, if $G$ acts irreducibly on $B, A \cap B$ must coincide with precisely one $G$-orbit of $A$, i.e., $A \cap B \in A / G$.

c) follows from Theorem 5.1 since $A / G$ is partition of $A$.

d) Let $\lambda: G \rightarrow \mathbb{C}$ be an eigenvalue of $G$ on $E$ and $0 \neq x \in E$ a corresponding eigenvector. Then $\lambda$ is a continuous homomorphism, and since $G$ is compact, $\lambda$ is unimodular. By c), one must have $y:=P_{F} x \neq 0$ for some $F \in A / G$, and by a), $y$ is also an eigenvector corresponding to $\lambda$.

Let $g \in G$ and $n:=|F|$, the length of the (induced) $G$-orbit $F$. Then $g^{n !}$ acts (induced) on $F$ as the identity. Hence, by Lemma 5.3, $g^{n !}$ acts (orginally) as the identity on $\operatorname{span}(F)$. This yields

$$
y=g^{n !} y=\lambda_{g}^{n !} y
$$

and hence $\lambda_{g}^{n !}=1$. As $g \in G$ was arbitrary, the eigenvalue $\lambda$ is torsion.

e) If $G$ acts irreducibly on $E$, then b) tells that $E$ is finite dimensional. As eigenvectors belonging to different eigenvalues have to be linearly independent, there can only finitely many eigenvalues, as claimed. 
Remark 5.5. For the special case of Banach sequence spaces, Theorem 5.4 has been first proved by de Jeu and Wortel in [JW14, Theorem 5.7].

We now shall list several criteria for the group $G$ in Theorem 5.4 to be trivial. In Section 6 we will translate those criteria into sufficient conditions for the strong convergence of positive operator semigroups.

A positive linear operator $T$ on a Banach lattice $E$ is called strongly positive if $T f$ is a quasi-interior point for every non-zero positive vector $f \in E$.

Corollary 5.6. Let $G$ be a strongly compact group of positive invertible operators on an atomic Banach lattice $E \neq\{0\}$. Then each one of the following assertions implies that $G=\left\{\mathrm{I}_{E}\right\}$ :

1) $G$ is divisible (cf. Appendix C).

2) $G$ has no clopen subgroups different from $G$.

3) $G$ contains a strongly positive operator.

4) Every finite-dimensional $G$-invariant band of $E$ on which $G$ acts irreducibly has dimension $\leq 1$.

5) $\mathbb{K}=\mathbb{C}$ and $G$ is Abelian and does not have any non-constant torsion eigenvalues.

Proof. By Lemma 5.3 it it suffices to prove in each of the mentioned cases that the group homomorphism $\varphi$, defined in (5.4), is trivial. We fix $a \in A$ and abbreviate $F:=\varphi_{G}(a)$.

1) $G$ acts transitively on $F$, which is a finite set. By a standard result from group theory, each homomorphism from a divisible group into a finite one must be trivial (see [GG17b, Lemma 2.3 and Proposition 2.4] for a proof). Hence $F=\{a\}$.

2) The set $H:=\left\{g \in G \mid \varphi_{g}(a)=a\right\}$ is a clopen subgroup of $G$ (since $A$ is discrete), so $H=G$.

3) Suppose that $g \in G$ is strongly positive. Then $\varphi_{g}(a)$ is a quasi-interior point and an atom, hence $\operatorname{dom}(E)=1$. In particular, $F=\{a\}$.

4) By Theorem [5.4, $\operatorname{span}(F)$ is a finite-dimensional $G$-invariant band of $E$ on which $G$ acts irreducibly. Hence $1 \leq|F|=\operatorname{dim}(\operatorname{span}(F)) \leq 1$, by assumption. If follows that $F=\{a\}$.

5) Let $m:=|F|$. We may consider $G$ as a compact Abelian group of $m \times m$-matrices acting on $\operatorname{span}(F) \cong \mathbb{C}^{m}$. Since $G$ is commutative, it is simultaneously diagonalizable. Each diagonal entry in a simultaneous diagonalization is an eigenvalue of $G$. By Theorem 5.4 such an eigenvalue is torsion, and hence, by asumption, trivial. This means that $G$ acts as the identity on $\operatorname{span}(F)$, which implies that $F=\{a\}$.

\section{Convergence of Positive Semigroups}

We shall now combine Theorem 4.3 with the findings of the previous section to obtain general results about strong convergence of positive operator semigroups. In all results of this section we shall take the following hypotheses (those of Theorem 4.3) as a starting point:

- $E$ is a Banach lattice;

- $T=\left(T_{s}\right)_{s \in S}$ is a positive and bounded operator semigroup on $E$; 
- $T$ restricts to a bounded semigroup on $E_{y}$ and is $E_{y}$-to- $E$ quasi-compact for some quasi-interior point $y \in E_{+}$.

Let us call these our standard assumptions for the remainder of this paper. The standard assumptions warrant that Theorem 4.3 is applicable, and we freely make use of this fact in the following.

Spectral-Theoretic Consequences. We first draw some spectral-theoretic conclusions.

Theorem 6.1. Suppose that an operator semigroup $\left(T_{s}\right)_{s \in S}$ on a complex Banach lattice $E$ satisfies the standard assumptions. Then the following assertions hold:

a) Each unimodular eigenvalue of $T$ is torsion.

b) If $T$ is irreducible, then it has only finitely many unimodular eigenvalues.

c) $T$ is strongly convergent if and only if $T$ has no non-constant torsion eigenvalue.

Proof. a) By Theorem 2.2, each unimodular eigenvalue of $T$ is the restriction of an eigenvalue of

$$
\mathcal{G}:=\left.\mathcal{T}\right|_{E_{\infty}},
$$

where $E_{\infty}=\left[\operatorname{ran}\left(P_{\infty}\right)\right]$. Since the latter space is atomic and $\mathcal{G}$ is compact, Theorem 5.4 yields that this eigenvalue is torsion.

b) If $T$ acts irreducibly on $E$ then, by Theorem $4.3, \mathcal{G}$ acts irreducibly on $\left[\operatorname{ran}\left(P_{\infty}\right)\right]$. Hence, $\mathcal{G}$ has only finitely many unimodular eigenvalues by Theorem 5.4. By Theorem 2.2. each eigenvalue of $T$ is the restriction of an eigenvalue of $\mathcal{G}$. This proves the claim.

c) The "if"-part follows from Corollary [5.6. For the "only if"-part we suppose that $T_{s} \rightarrow P$ is strongly convergent. Then $P=P_{\infty}$ and $\mathcal{G}=\left\{\mathrm{I}_{E_{\infty}}\right\}$. Since each unimodular eigenvalue of $T$ is the restriction of an eigenvalue of $\mathcal{G}$ (Theorem 2.2), $T$ has no non-constant unimodular eigenvalues.

Sufficient Conditions for Convergence. Apart from the spectral characterization of the previous theorem, Corollary 5.6 yields the following sufficient conditions for the convergence of a positive semigroup.

Theorem 6.2. Suppose that an operator semigroup $\left(T_{s}\right)_{s \in S}$ on a Banach lattice E satisfies the standard assumptions. In addition, let at least one of the following conditions be satisfied:

1) $S$ is essentially divisible (e.g.: $S$ is divisible or generates a divisible group; cf. Appendix (C);

2) $S$ carries a topology such that $T$ is strongly continuous and the only clopen subsemigroup of $S$ containing 0 is $S$ itself (e.g.: $S$ is connected).

3) $T_{s}$ is strongly positive for some $s \in S$.

Then $T$ is strongly convergent.

Proof. Theorem 4.3 is applicable, so it suffices to consider the case that $E \neq\{0\}$ is atomic and has order-continuous norm and that $\mathcal{T}$ is a compact group of positive invertible operators on $E$. We must show that $\mathcal{T}$ acts trivially on $E$.

1) By Corollary 5.6 it suffices to show that $\mathcal{T}$ is divisible. But this follows from a straightforward compactness argument. 
2) Let $\mathcal{H}$ be any clopen subgroup of $\mathcal{T}$. Then $H:=\left\{s \in S \mid T_{s} \in \mathcal{H}\right\}$ is a clopen subsemigroup of $S$. (Note that $H \neq \emptyset$ since $\mathcal{H} \neq \emptyset$ is open and $T_{S}$ is dense in $\mathcal{T}$.) By hypothesis, $H=S$, so $T_{S} \subseteq \mathcal{H}$. Since $\mathcal{H}$ is closed and $T_{S}$ is dense in $\mathcal{T}$, it follows that $\mathcal{H}=\mathcal{T}$. Hence, $\mathcal{T}=\left\{\mathrm{I}_{E}\right\}$ by Corollary 5.6.

3) If $T_{s}$ is strongly positive, then $\mathcal{T}$ contains a strongly positive operator, and we conclude with the help of Corollary 5.6.

Remarks 6.3. 1) Condition 1) is satisfied, in particular, if $S=\mathbb{R}_{+}$(divisible semigroup), but also if $S=\{0\} \cup[1, \infty)$ (not divisible, but generating a divisible group). Note that in the latter case, the semigroup direction is just a subordering of the natural one, but does not coincide with it. Nevertheless, the associated notions of "limit" do coincide.

2) Condition 1) is also satisfied when $S=[0, \infty)$, endowed with the semigroup operation $(a, b) \mapsto a \vee b=\max \{a, b\}$. The semigroup direction coincides with the natural ordering. This semigroup is neither divisible nor does it generate a divisible group (it is not even cancellative). However, it is essentially divisible.

On the other hand, this example is a little artificial, as each element of $S$ is an idempotent, and hence a representation $T=\left(T_{s}\right)_{s}$ is just a family of projections with decreasing ranges as $s$ increases. For such semigroups, the question of convergence can often be treated by other methods.

3) The semigroup of positive dyadic rationals is

$$
D_{+}:=\{0\} \cup\left\{\frac{k}{2^{n}} \mid k, n \in \mathbb{N}_{0}\right\} .
$$

The semigroup direction on $D_{+}$coincides with the usual ordering. It is easy to see that $D_{+}$is not essentially divisible. If we endow $D_{+}$with its natural topology, $D_{+}$is not connected. However, $D$ is the only clopen subsemigroup of $D_{+}$containing 0. (Actually, apart from $D_{+}$itself there is no other open subsemigroup of $D_{+}$containing 0 .)

Hence, from Theorem 6.2 it follows that each strongly continuous representation of $D_{+}$that satisfies the standard hypotheses is strongly convergent. Without strong continuity, however, this can fail. In fact, let $D=D_{+}-D_{+}$ denote the group generated by $D_{+}$in the real numbers. Then $D$ has a subgroup of index 3 (namely $3 D$ ), so by the same construction as in the proof of [GG17b, Theorem 2.5] we can find a positive and bounded representation of $(D,+)$ on the Banach lattice $\mathbb{R}^{3}$. The restriction of this representation to $\left(D_{+},+\right)$satisfies the standard conditions, but it does not converge.

4) With Theorem 6.2, Condition 3), we generalize a result of Gerlach, cf. Ger13, Theorem 4.3].

Lattice Subrepresentations. A closed linear subspace $F$ of Banach lattice $E$ is called a lattice subspace, if it is a Banach lattice with the order induced by $E$ but with respect to an equivalent norm. A lattice subspace need not be a sublattice. (By Theorem 4.1, the range of a positive projection is always a lattice subspace.)

Given a representation $T=\left(T_{s}\right)_{s \in S}$ on a Banach lattice $E$, each $T$-invariant lattice subspace gives rise to a lattice subrepresentation. So the lattice subrepresentations are those subrepresentations where the underlying space is a lattice subspace. 
Theorem 6.4. Suppose that an operator semigroup $T=\left(T_{s}\right)_{s \in S}$ on a Banach lattice $E$ satisfies the standard assumptions. In addition, suppose that each finitedimensional lattice subrepresentation of $T$ is at most one-dimensional. Then $T$ is strongly convergent.

Proof. As in the proof of Theorem 6.2 it suffices to consider the case that $E \neq\{0\}$ is atomic and that $\mathcal{T}$ is a compact group of positive invertible operators on $E$. It then follows that each finite-dimensional $T$-invariant band of $E$ is at most onedimensional. Corollary 5.6 is applicable and yields the claim.

\section{Conclusion: Some Classical Theorems Revisited}

In this section we start with a little historical survey and end with demonstrating how our approach leads to far-reaching generalizations of the "classical" results.

Historical Note. In 1982, Günther Greiner in the influential paper Gre82 proved the following result as "Corollary 3.11":

Theorem 7.1 (Greiner 1982). Let $T=\left(T_{s}\right)_{s \geq 0}$ be a positive contraction $C_{0}$ semigroup on a space $E=\mathrm{L}^{p}(\mathrm{X}), 1 \leq p<\infty$, with the following properties:

1) There is a strictly positive $T$-fixed vector;

2) For some $s_{0}>0$ the operator $T_{s_{0}}$ is a kernel operator.

Then $\lim _{s \rightarrow \infty} T_{s} x$ exists for each $x \in E$.

For the proof, Greiner employed what has become known as "Greiner's 0/2-law" (see [Gre82, Theorem 3.7] and also [GN82]) and a result of Axmann from [Axm80. Both results have involved proofs and make use of the lattice structure on the regular operators on Banach lattices with order-continuous norm. The relevance of Greiner's theorem derives from the fact that the assumptions can be frequently verified for semigroups arising in partial differential equations or in stochastics.

For a long time, Greiner's theorem stood somehow isolated within the asymptotic theory of (positive) semigroups. The "revival" of Greiner's theorem as a theoretical result began with a paper of Davies Dav05 from 2005. Davies showed that the peripheral point spectrum of the generator $A$ of a $C_{0}$-semigroup $T$ of positive contractions on a space $E=\mathrm{L}^{p}(\mathrm{X}), 1 \leq p<\infty$, has to be trivial in the following cases: (1) X is countable with the counting measure and (2) $\mathrm{X}$ is locally compact and second countable and $T$ has the Feller property (i.e., each $T_{s}$ for $s>0$ maps $E$ into the space of continuous functions). Case (1) was subsequently generalized by Keicher in Kei06 to bounded and positive $C_{0}$-semigroups on atomic Banach lattices with order-continuous norm, and by Wolff Wol08 to more general atomic Banach lattices.

Shortly after, Arendt in Are08 generalized Davies' results towards the following theorem.

Theorem 7.2 (Greiner 1982/Arendt 2008). Let $A$ be the generator of a positive contraction $C_{0}$-semigroup $T=\left(T_{s}\right)_{s \geq 0}$ on a space $E=\mathrm{L}^{p}(\mathrm{X}), 1 \leq p<\infty$. Suppose that for some $s_{0}>0$ the operator $T_{s_{0}}$ is a kernel operator. Then $\sigma_{\mathrm{p}}(A) \cap \mathrm{i} \mathbb{R} \subseteq\{0\}$.

This result is "Theorem 3.1" in Arendt's paper Are08. Interestingly, as observed by Gerlach in Ger13, it already appears in Greiner's 1982 paper, namely in the first paragraph of his proof of Theorem 7.1 (i.e., his "Corollary 3.11"). We 
will thus call Theorem 7.2 the Greiner-Arendt theorem. Arendt points out that Theorem 7.2 implies Davies' result: in case (1) every positive operator is a kernel operator, whereas in case (2) the Feller property implies that each $T_{s}$ for $s>0$ is a kernel operator. (This follows from Bukhvalov's characterization of kernel operators, cf. [Are08, Corollary 2.4].)

Let us briefly sketch Arendt's proof of Theorem 7.2 If $f \in E$ is an eigenvector of $A$ for the eigenvalue $\lambda \in i \mathbb{R}$, then $T_{s}|f| \geq|f|$ for all $s \geq 0$. Since each $T_{s}$ is a contraction and the norm on $\mathrm{L}^{p}$ is strictly monotone, it follows that $|f|$ is a fixed point. By restricting to the set $[|f|>0]$ one can assume that $|f|$ is strictly positive. Next, from the weak compactness of the order interval $[0,|f|]$ it follows that the semigroup is weakly relatively compact. Then the JdLG-theory enters the scene and reduces to problem to an atomic Banach lattice with order continuous norm. Finally, Keicher's analysis from [Kei06] shows that the dynamics there must be trivial, and hence $\lambda=0$.

Arendt's paper is remarkable in several respects. First of all, his proof of Theorem 7.2 employs the JdLG-theory which is central also to the more recent work of Gerlach and Glück, and to the present paper. Secondly, Arendt recalls Greiner's Theorem 7.1 and gives a proof (building, as Greiner did, on Theorem 7.2) under the additional assumption that the semigroup is irreducible. (This proof appears to be the first complete one in English language, cf. AAre08, Remark 4.3].) Thirdly, Arendt promotes Greiner's result by illustrating its use with several concrete examples.

Most remarkable of all, however, is what is not written in Are08: namely that Greiner's Theorem 7.1 almost directly implies the Greiner-Arendt Theorem 7.2 Indeed, one starts exactly as in Arendt's proof until one has found the quasiinterior fixed point $|f|$; then Greiner's theorem tells that $\lim _{s \rightarrow \infty} T_{s} f$ exists, and hence $\lambda=0$ follows.

In the following years the topic was taken up by M. Gerlach and J. Glück. Gerlach Ger13 discussed Greiner's approach in a general Banach lattice setting and extended it to semigroups that merely dominate a kernel operator; he also noted that the dominated kernel operator can be replaced by a compact operator. In their quest to find a unifying framework, and stimulated by "Corollary 3.8" in Keicher's paper [Kei06, Gerlach and Glück in GG17a, GG17b finally identified "AM-compactness" as the right property generalizing the different cases. Alongside a unification, this led also to a major simplification, since AM-compactness is much more easily shown directly than by passing through the concept of a kernel operator. (E.g., it follows directly from Theorem A.4 that a Feller operator as considered by Davies is AM-compact.)

Finally, Gerlach and Glück realized that strong continuity of the semigroup can be dispensed with, since arguments requiring time regularity can be replaced by purely algebraic ones. This led to proofs for most of the above-mentioned results for semigroups without any time regularity.

Somewhat independently from the above development, Pichór and Rudnicki proved convergence results for Markov semigroups which merely dominate a nontrivial kernel operator [PR00, Theorems 1 and 2]. Their results are closely related to (and earlier than) the results of Gerlach Ger13, but their approach is different, focusing on $L^{1}$-spaces and employing methods from stochastics. Later on, in PR16, PR18, these authors adapted their original results to various situations 
involving semigroups on $L^{1}$-spaces, with numerous applications in mathematical biology.

Remark 7.3. As noted above, the implication "Theorem $7.1 \Rightarrow$ Theorem [7.2' is almost immediate. Now, in hindsight, it becomes clear that Arendt in Are08 was also very close to proving the converse implication "Theorem $7.2 \Rightarrow$ Theorem 7.1'. Indeed, the weak compactness of order intervals in $\mathrm{L}^{p}$-spaces for $1 \leq p<\infty$ implies that on such spaces a positive bounded semigroup $T=\left(T_{s}\right)_{s \geq 0}$ with a quasi-interior fixed point is relatively weakly compact. Hence, the "triviality of the peripheral point spectrum" asserted by Theorem 7.2 implies that $T$ acts trivially on the "reversible" part of the corresponding JdLG-decomposition. One can then infer strong convergence of $T$ if one knows that $T$ is not just relatively weakly, but even relatively strongly compact. And the latter holds, in fact, since kernel operators are AM-compact; but in this context this was noted only later by Gerlach and Glück.

Old Theorems in a New Light. Let us now review some of the abovementioned results in the light of our actual findings. First of all, consider the following result, which is merely an instantiation of Theorem 6.2 a), to the (divisible!) semigroup $\mathbb{R}_{+}$.

Theorem 7.4. Let $T=\left(T_{s}\right)_{s \geq 0}$ be a positive and bounded (but not necessarily strongly continuous) semigroup on a Banach lattice $E$ with the following properties:

1) There is a quasi-interior point $y \in E_{+}$and $c>0$ such that $T_{s} y \leq c y$ for all $s \geq 0$.

2) For some $s_{0}>0$ the operator $T_{s_{0}}$ is AM-compact.

Then $\lim _{s \rightarrow \infty} T_{s} x$ exists for each $x \in E$.

Theorem 7.4 is a slight strengthening of Theorem 4.5 from GG17b, where the quasi-interior point $y$ is required to be $T$-fixed. It implies Greiner's Theorem 7.1 as a special case: simply note that on $E:=\mathrm{L}^{p}(\mathrm{X})$ a strictly positive function is quasi-interior and that a kernel operator is AM-compact (Theorem A.3).

In the next result, the requirement that one of the semigroup operators is AMcompact is relaxed towards a mere domination property, however on the expenses of strengthening other hypotheses.

Theorem 7.5. Let $T=\left(T_{s}\right)_{s \geq 0}$ be a positive, bounded and irreducible (but not necessarily strongly continuous) semigroup on a Banach lattice $E$ with order continuous norm. Suppose that the conditions are satisfied:

1) There is a quasi-interior point $y \in E_{+}$such that $T_{s} y \leq y$ for all $s \geq 0$.

2) For some $s_{0}>0$ there is an AM-compact operator $K \neq 0$ with $0 \leq K \leq T_{s_{0}}$.

Then $\lim _{s \rightarrow \infty} T_{s} x$ exists for each $x \in E$.

Proof. By Lemma 4.5 $T$ satisfies the standard assumptions (see Section 6). Hence, as $\mathbb{R}_{+}$is a divisible semigroup, the assertions follow from Theorem 6.2 a).

Theorem 7.5 is a generalization of the abovementioned results PR00, Theorems 1 and 2] of Pichór und Rudnicki for stochastic $C_{0^{-}}$-semigroups on $L^{1}$-spaces. For $C_{0^{-}}$ semigroups on Banach lattices, the theorem is due to Gerlach Ger13, Theorem 4.2]. 
We note that irreducibility of the semigroup can be replaced by other assumptions ensuring that the AM-compact operator $K$ is "sufficiently large" when compared with the semigroup. A very general result of this type was proved by Gerlach and Glück in [GG17b, Theorem 3.11].

Let us finally return to the spectral-theoretic results (by Davies, Keicher, Wolff and Greiner-Arendt) discussed above. In this direction, we establish the following general theorem.

Theorem 7.6. Let $T=\left(T_{s}\right)_{s \in S}$ be a bounded and positive semigroup on a Banach lattice $E$. Suppose that for some $s \in S$ the operator $T_{s}$ is AM-compact. Then the following assertions hold:

a) Each unimodular eigenvalue is torsion.

b) If $T$ is irreducible then there are only finitely many unimodular eigenvalues.

c) Suppose that 1) $S$ is essentially divisible or 2) $T$ is strongly continuous with respect to some topology on $S$ such that the only clopen subsemigroup of $S$ containing 0 is $S$ itself. Then the only possible unimodular eigenvalue of $T$ is the constant one.

Proof. We combine the classical ideas from Scheffold [Sch71] as employed by Keicher in [Kei06, Theorem 3.1] with the theory developed in this paper.

a) Let $\lambda=\left(\lambda_{s}\right)_{s \in S}$ be a unimodular eigenvalue of $T$, and let $0 \neq z \in E$ be a corresponding eigenvector. Abbreviate $y:=|z| \in E_{+}$. Then

$$
0 \neq y=|z|=\left|\lambda_{s} z\right|=\left|T_{s} z\right| \leq T_{s}|z|=T_{s} y \quad(s \in S) .
$$

It follows that the net $\left(T_{s} y\right)_{s \in S}$ is increasing.

One can find a positive linear functional $\varphi \in E_{+}^{\prime}$ such that $\varphi(y)>0$. Define

$$
J:=\left\{x \in E \mid \lim _{s \in S} \varphi\left(T_{s}|x|\right)=0\right\} .
$$

It is routine to check that $J$ is a closed and $T$-invariant ideal. Since $\varphi\left(T_{s} y\right) \geq$ $\varphi(y)>0$ for all $s \in S$, we have $y \notin J$. Moreover, $T_{t} y-y \in J$ since

$$
\varphi\left(T_{s}\left|T_{t} y-y\right|\right)=\varphi T_{s}\left(T_{t} y-y\right)=\varphi\left(T_{t+s} y\right)-\varphi\left(T_{s} y\right) \quad(s \in S)
$$

and $\left(\varphi\left(T_{s} x\right)\right)_{s \in S}$ is increasing and bounded.

Since $J$ is a closed $T$-invariant ideal, the quotient space $E_{1}:=E / J$ naturally carries the structure of a Banach lattice, and the representation $T$ on $E$ induces a representation $\hat{T}$ on $E_{1}$ by

$$
\hat{T}_{s}(x+J):=T_{s} x+J \quad(s \in S, x \in E) .
$$

Let $\hat{z}:=z+J$ and $\hat{y}:=y+J$ be the equivalence classes of $z$ and $y$ in $E_{1}=E / J$, respectively. Since the canonical surjection is a lattice homomorphism, $\hat{y}=|\hat{z}|$ in $E_{1}$. Since $y \notin J, \hat{z} \neq 0$. It follows that $\hat{z}$ is an eigenvector of $\hat{T}$ for the eigenvalue $\lambda$.

Since $T_{s} y-y \in J$ for each $s \in S$, the point $\hat{y}$ is $T$-fixed for the induced semigroup on $E_{1}$. Moreover, by the hypothesis and Theorem A.2, for some $s \in S$ the operator $\hat{T}_{s}$ is AM-compact. Hence, when we restrict to the closed ideal $E_{2}:=\overline{F_{\hat{y}}}$ generated by $\hat{y}$ in $E_{1}$, we find that the semigroup $\hat{T}$ restricted to $E_{2}$ satisfies the standard assumptions. Theorem 6.1 then yields that that $\lambda$ must be torsion. 
c) We start again as in the proof of a). By Theorem 6.2, either condition 1) and 2) implies that $\hat{T}$ on $E_{2}$ is convergent. Then, by Theorem 6.1, we conclude that $\lambda$ is constant.

Theorem 7.6 generalizes the Greiner-Arendt Theorem 7.2, simply specialize $S=\mathbb{R}_{+}$and note that kernel operators are AM-compact (Theoren A.3). A fortiori, it generalizes Davies' results from Dav05. However, it also implies Keicher's result Kei06, Theorem 3.1] (but not Wolff's), as on an atomic Banach lattice with ordercontinuous norm all order intervals are relatively compact, and hence all bounded operators are AM-compact. Finally, Theorem 7.6 also generalizes GG17b, Theorem 4.19].

\section{Appendix A. AM-COMpaCt Operators}

Let $E$ be a Banach lattice and $F$ a Banach space. A bounded operator $T: E \rightarrow F$ is called AM-compact if $T$ maps order intervals of $E$ to relatively compact subsets of $F$.

The following is a useful characterization of AM-compactness in the case that $E$ has a quasi-interior point $y \in E_{+}$. Recall that this means that the principal ideal

$$
E_{y}:=\{x \in E \mid \text { there is } c \geq 0 \text { such that }|x| \leq c y\}
$$

is dense in $E$. We endow $E_{y}$ with its natural AM-norm

$$
\|x\|_{y}:=\inf \{c \geq 0|| x \mid \leq c y\} .
$$

It is well known that this turns $E_{y}$ into a Banach lattice, isometrically lattice isomorphic to $\mathrm{C}(K)$ (with $y$ being mapped to $\mathbf{1}$ ) for some compact Hausdorff space $K$ (Krein-Krein-Kakutani theorem).

Lemma A.1. Let $E$ be a Banach lattice with a quasi-interior point $y \in E_{+}$, let $F$ be any other Banach space and $T: E \rightarrow F$ a bounded operator. Then $T: E_{y} \rightarrow F$ is compact if and only if $T[0, y]$ is relatively compact, if and only if $T$ is AM-compact.

Proof. Suppose that $T: E_{y} \rightarrow F$ is compact. Then, $T[0, y]$ is relatively compact. If the latter is the case, then for each $c>0$ the set

$$
T[-c y, c y]=T(-c y+2 c[0, y])=T(-c y)+2 c T[0, y]
$$

is also relatively compact. Let $u \in E_{+}$and $\varepsilon>0$. Since $y$ is a quasi-interior point, there is $c>0$ such that $u \in[-c y, c y]+\mathrm{B}[0, \varepsilon]$. We claim that

$$
[0, u] \subseteq[-c y, c y]+\mathrm{B}[0, \varepsilon]
$$

as well. Indeed, write $u=z+r$ with $|z| \leq c y$ and $\|r\| \leq \varepsilon$ and let $0 \leq x \leq u$. Then $0 \leq x \leq|z|+|r| \leq c y+|r|$. By the decomposition property, there are $0 \leq x_{1} \leq|z|$ and $0 \leq x_{2} \leq|r|$ with $x=x_{1}+x_{2}$. Hence $x \in[-c y, c y]+\mathrm{B}[0, \varepsilon]$ as claimed.

It follows from (A.1) that

$$
T[0, u] \subseteq T[-c y, c y]+\mathrm{B}[0,\|T\| \varepsilon] .
$$

Since $T[-c y, c y]$ is relatively compact, it admits a finite $\|T\| \varepsilon$-mesh. Hence, $T[0, u]$ admits a finite $2\|T\| \varepsilon$-mesh. As $\varepsilon>0$ was arbitrary, $T[0, u]$ is relatively compact.

Finally, if $[u, v]$ is any non-empty order interval of $E$, then $v-u \geq 0$ and $T[u, v]=T u+T[0, v-u]$ is relatively compact, by what we have already shown. 
The following theorem shows that AM-compactness is preserved when one passes to a factor lattice with respect to an invariant closed ideal.

Theorem A.2. Let $E$ be a Banach lattice, let $T \in \mathcal{L}(E)$ be AM-compact, and let $I \subseteq E$ be a $T$-invariant closed lattice ideal in $E$. Then the induced operator

$$
T_{/}: E / I \rightarrow E / I \quad T_{/}(x+I):=T x+I \quad(x \in E)
$$

is also AM-compact.

Proof. Let $0 \leq X \in E / I$. We have to show: the order interval $[0, X]$ in $E / I$ is mapped by $T_{\text {/ }}$ to a relatively compact subset of $E / I$.

Recall from [Sch74, Prop. II.2.6] that for $x, y \in E$ one has

$$
x+I \leq y+I \quad \Longleftrightarrow \quad \exists x_{1}, y_{1} \in E: x-x_{1}, y-y_{1} \in I, x_{1} \leq y_{1} .
$$

In particular, there is $x \geq 0$ such that $X=x+I$. Now, let $0 \leq Y \leq X$ be given. Then there are $y \in E$ and $z \in I$ such that $y \leq x+z$ and $Y=y+I$. Replacing $z$ by $|z|$ we may suppose in addition that $z \geq 0$.

By the decomposition property, $y^{+}=a+b$ for some elements $0 \leq a \leq x$ and $0 \leq b \leq z$. Then $b \in I$ since $I$ is an ideal and $z \in I$. As the canonical surjection is a lattice homomorphism, $Y^{+}=a+I$ and hence $T_{/}\left(Y^{+}\right)=T a+I$. Similarly, one can show that there is $c \in[0, x]$ with $Y^{-}=c+I$. It follows that

$$
T_{/}(Y)=T_{/}\left(Y^{+}\right)-T_{/}\left(Y^{-}\right) \in T([-x, x])+I
$$

and hence $T_{/}([0, X]) \subseteq T([-x, x])+I$. Since $T$ is AM-compact, the set $T([-x, x])$ is relatively compact in $E$, and hence so is $T([-x, x])+I$ in $E / I$. This implies that $T_{/}([0, X])$ is relatively compact, as desired.

Examples of AM-Compact Operators. In the remainder of this appendix we consider two results that help to identify AM-compact operators. The first tells that every integral operator is AM-compact. This is a well-known consequence of abstract theory (see e.g. the discussion at the beginning of [GG17b, Section 4], which is based on abstract results in [MN91, Corollary 3.7.3] and Sch74, Proposition IV.9.8]). Gerlach and Glück give an elementary proof involving measuretheoretic (i.e., almost everywhere) arguments GG17b, Proposition A.1]. Our proof is a little less elementary, but replaces the measure theory by functional analysis.

Theorem A.3. Let $\mathrm{X}$ and $\mathrm{Y}$ be measure spaces and let $1 \leq p, q<\infty$. Let, furthermore, $k: X \times Y \rightarrow \mathbb{R}_{+}$be a measurable mapping such that by

$$
T f(x):=\int_{\mathrm{Y}} k(x, \cdot) f \quad(x \in X)
$$

a bounded operator

$$
T: \mathrm{L}^{p}(\mathrm{Y}) \rightarrow \mathrm{L}^{q}(\mathrm{X})
$$

is (well) defined. Then $T$ is AM-compact.

Proof. Let the measure spaces be $\mathrm{X}=\left(X, \Sigma_{\mathrm{X}}, \mu_{\mathrm{X}}\right)$ and $\mathrm{Y}=\left(Y, \Sigma_{\mathrm{Y}}, \mu_{\mathrm{Y}}\right)$. Let $0 \leq u \in \mathrm{L}^{p}(\mathrm{Y})$. We have to show that $T[0, u] \subseteq \mathrm{L}^{q}(\mathrm{X})$ is relatively compact.

Suppose first that both measures $\mu_{\mathrm{X}}$ and $\mu_{\mathrm{Y}}$ are finite, $u=\mathbf{1}$ and $T \mathbf{1} \in \mathrm{L}^{\infty}(\mathrm{X})$. Then, in particular, $k \in \mathrm{L}^{1}(\mathrm{X} \times \mathrm{Y})$. Since $\mathrm{L}^{1}(\mathrm{X}) \otimes \mathrm{L}^{1}(\mathrm{Y})$ is dense in $\mathrm{L}^{1}(\mathrm{X} \times \mathrm{Y})$, $T: \mathrm{L}^{\infty}(\mathrm{Y}) \rightarrow \mathrm{L}^{1}(\mathrm{X})$ is compact. But $T$ factors through $\mathrm{L}^{\infty}(\mathrm{X})$ (since $T \mathbf{1}$ is bounded) and since on closed $\mathrm{L}^{\infty}$-balls the $\mathrm{L}^{q}$ - and the $\mathrm{L}^{1}$-topology coincide, $T$ : $\mathrm{L}^{\infty}(\mathrm{Y}) \rightarrow \mathrm{L}^{q}(\mathrm{X})$ is compact. In particular, $T[0, \mathbf{1}]$ is relatively compact in $\mathrm{L}^{q}(\mathrm{X})$. 
In the general case, consider the operator

$$
S: \mathrm{L}^{p}\left(Y, \Sigma_{\mathrm{Y}}, u^{p} \mu_{\mathrm{Y}}\right) \rightarrow \mathrm{L}^{q}(\mathrm{X}), \quad S f:=T(u f) .
$$

Because of $[0, u]=u \cdot[0, \mathbf{1}]$ it suffices to show that $S[0, \mathbf{1}] \subseteq \mathrm{L}^{q}(\mathrm{X})$ is relatively compact. Hence, one may suppose without loss of generality that $\mu_{\mathrm{Y}}$ is finite and $u=1$.

Under this assumption let

$$
g=T \mathbf{1}:=\left(x \mapsto \int_{Y} k(x, y) \mu_{\mathrm{Y}}(\mathrm{d} y)\right) \in \mathrm{L}^{q}(\mathrm{X})
$$

and, for $n \in \mathbb{N}$,

$$
g_{n}:=\mathbf{1}_{\left[\frac{1}{n} \leq g \leq n\right]} g \in \mathrm{L}^{1}(\mathrm{X}) \cap \mathrm{L}^{\infty}(\mathrm{X})
$$

Define $T_{n}$ by

$$
T_{n} f:=\mathbf{1}_{\left[\frac{1}{n} \leq g \leq n\right]} T f=\int_{Y} \mathbf{1}_{\left[\frac{1}{n} \leq g \leq n\right]}(x) k(x, y) f(y) \mu_{\mathrm{Y}}(\mathrm{d} y) .
$$

Observe that $T_{n} \mathbf{1} \in \mathrm{L}^{\infty}(\mathrm{X})$ and it is supported on a set of finite measure. So, by what we have shown first, $T_{n}: \mathrm{L}^{\infty}(\mathrm{Y}) \rightarrow \mathrm{L}^{q}(\mathrm{X})$ is compact. Now,

$$
\left|\left(T-T_{n}\right) f\right| \leq\left(\mathbf{1}_{\left[g \leq \frac{1}{n}\right]}+\mathbf{1}_{[g \geq n]}\right) g\|f\|_{L^{\infty}}
$$

and hence

$$
\left\|T-T_{n}\right\|_{\mathrm{L}^{q} \leftarrow \mathrm{L}^{\infty}} \leq\|\ldots g\|_{\mathrm{L}^{q}} \rightarrow 0 \quad(n \rightarrow \infty) .
$$

Consequently, also $T: \mathrm{L}^{\infty}(\mathrm{Y}) \rightarrow \mathrm{L}^{q}(\mathrm{X})$ is compact, and this concludes the proof.

We turn to a second class of examples of AM-compact operators.

Theorem A.4. Let $\mathrm{X}$ and $\mathrm{Y}$ be measure spaces, let $1 \leq p, q<\infty$, and let

$$
T: \mathrm{L}^{p}(\mathrm{Y}) \rightarrow \mathrm{L}^{q}(\mathrm{X})
$$

be a positive operator with the following property: There is a sequence $\left(A_{n}\right)_{n}$ in $\Sigma_{\mathrm{X}}$ such that $\bigcup_{n} A_{n}=X$ and such that

$$
\mathbf{1}_{A_{n}} \cdot \operatorname{ran}(T) \subseteq \mathrm{L}^{\infty}\left(A_{n}\right) \quad \text { for all } n \in \mathbb{N} .
$$

Then $T$ is AM-compact.

Proof. By performing the same reduction as in the proof of Theorem A.3 above, we may suppose without loss of generality that $\mathrm{Y}$ is a finite measure space. It remains to show that $T[0, \mathbf{1}]$ is relatively $\mathrm{L}^{q}$-compact.

Define the projection $P_{n}$ on $\mathrm{L}^{q}(\mathrm{X})$ by

$$
P_{n} f:=\mathbf{1}_{A_{n} \cap\left[T \mathbf{1} \geq \frac{1}{n}\right]} f .
$$

By hypothesis and the dominated convergence theorem, $P_{n} T \rightarrow T$ uniformly on order intervals of $\mathrm{L}^{p}(\mathrm{Y})$, hence in particular with respect to the norm of $\mathcal{L}\left(\mathrm{L}^{\infty} ; \mathrm{L}^{q}\right)$. Therefore, it suffices to consider the case that also $\mathrm{X}$ is a finite measure space, and $\operatorname{ran}(T) \subseteq \mathrm{L}^{\infty}(\mathrm{X})$. By the subsequent Lemma A.5 we are done.

Lemma A.5. Let $\mathrm{X}$ and $\mathrm{Y}$ be finite measure spaces, $1 \leq p, q<\infty$ and $T: \mathrm{L}^{p}(\mathrm{Y}) \rightarrow$ $\mathrm{L}^{q}(X)$ a bounded operator with $\operatorname{ran}(T) \subseteq \mathrm{L}^{\infty}(\mathrm{X})$. Then for each $1<r \leq \infty$ with $r \geq p$ the operator

$$
\left.T\right|_{\mathrm{L}^{r}}: \mathrm{L}^{r}(\mathrm{Y}) \rightarrow \mathrm{L}^{q}(\mathrm{X})
$$

is compact. 
Proof. We may suppose $1<p=r<\infty$. By the closed graph theorem, $T: \mathrm{L}^{p}(\mathrm{Y}) \rightarrow$ $\mathrm{L}^{\infty}(\mathrm{X})$ is bounded. If $\left(f_{n}\right)_{n}$ is a bounded sequence in $\mathrm{L}^{p}(\mathrm{Y})$, it has a weakly convergent subsequence (by reflexivity). Hence, $\left(T f_{n}\right)_{n}$ has a weakly convergent subsequence in $\mathrm{L}^{\infty}(\mathrm{X})$. But a weakly convergent sequence in $\mathrm{L}^{\infty}(\mathrm{X})$ converges strongly in $\mathrm{L}^{q}(\mathrm{X})$ since $\mathrm{X}$ is a finite measure space. (Represent $\mathrm{L}^{\infty}(\mathrm{X}) \cong \mathrm{C}(K)$ for some compact Hausdorff space $K$, and observe that in $\mathrm{C}(K)$ a sequence(!) is weakly convergent if and only if it is uniformly bounded and pointwise convergent.)

Remarks A.6. 1) Actually, one can reduce Theorem A.4 to Theorem A.3 by employing Bukhvalov's characterization of integral operators Buk78, variants of which are for instance discussed in [GvE81, Theorem 3.9], Zaa83, Theorem 96.5], [AB94, Theorem 1.5] and [Ger14, Theorem 4.2.12]

2) For $1 \leq p \leq 2$, Lemma A.5 has a much more elementary proof. Indeed, by quite elementary arguments one can show that $T$ is a Hilbert-Schmidt operator when considered as an operator $\mathrm{L}^{2}(\mathrm{Y}) \rightarrow \mathrm{L}^{2}(\mathrm{X})$.

3) In the proof of Lemma A.5 we have used that, for a finite measure space $\mathrm{X}$, a sequence which converges weakly in $\mathrm{L}^{\infty}(\mathrm{X})$ must converge strongly on $\mathrm{L}^{q}(\mathrm{X})$ for each $1 \leq q<\infty$. It would be nice to have a more elementary proof of this fact, avoiding the representation $\mathrm{L}^{\infty}(\mathrm{X}) \cong \mathrm{C}(K)$.

\section{Appendix B. Universal Nets}

In this appendix we treat, for the convenience of the reader, a useful but maybe not so widely known concept from general topology.

Let $(I, \leq)$ be any directed set. A subset $A$ of $I$ is called a tail of $I$ if it is of the form $A=\left\{\alpha \in I \mid \alpha \geq \alpha_{0}\right\}$ for some $\alpha_{0} \in I$. And it is called cofinal if its complement does not contain a tail. Equivalently, $A$ is cofinal if for each $\alpha_{0} \in I$ there is $\alpha \geq \alpha_{0}$ such that $\alpha \in A$. Clearly, each tail is cofinal.

A tail of a net $\left(x_{\alpha}\right)_{\alpha \in I}$ in a set $X$ is a subset of the form $\left\{x_{\alpha} \mid \alpha \in A\right\}$, where $A \subseteq I$ is a tail of $I$. A net $\left(x_{\alpha}\right)_{\alpha}$ is called universal or an ultranet, if for each $Y \subseteq X$ either the set $Y$ or its complement contains a tail of the net. If $f: X_{1} \rightarrow X_{2}$ is a mapping and $\left(x_{\alpha}\right)_{\alpha}$ is a universal net in $X_{1}$, then $\left(f\left(x_{\alpha}\right)\right)_{\alpha}$ is a universal net in $X_{2}$. Likewise, a subnet of a universal net is universal.

Lemma B.1. Each net has a universal subnet.

Proof. Let $\left(x_{\alpha}\right)_{\alpha}$ be a net in a set $X$. Then the tails of the net form a filter base, hence by Zorn's lemma there is an ultrafilter $\mathcal{U}$ containing all the tails. For $\beta \in I$ and $U \in \mathcal{U}$ there is $\alpha(\beta, U) \in I$ such that $\alpha(\beta, U) \geq \beta$ and $x_{\alpha(\beta, U)} \in U$. The set $I \times \mathcal{U}$ is directed by

$$
(\alpha, U) \leq(\beta, V) \stackrel{\text { def. }}{\Longleftrightarrow} \alpha \leq \beta \wedge V \subseteq U .
$$

The net $\left(x_{\alpha(\beta, U)}\right)_{\beta, U}$ is a universal subnet of $\left(x_{\alpha}\right)_{\alpha}$.

Since a universal net in a topological space $X$ converges if and only if it has a convergent subnet, $X$ is compact if and only if each universal net in $X$ converges.

Lemma B.2. Let $X$ be a metric space and let $\left(x_{\alpha}\right)_{\alpha \in I}$ be a net in $X$. Consider the following three assertions:

(i) For each $\varepsilon>0$ there is a compact set $K \subseteq X$ such that $\mathrm{B}[K, \varepsilon]$ contains a tail of $\left(x_{\alpha}\right)_{\alpha}$. 
(ii) For each $\varepsilon>0$ there is $z \in X$ such that $\left\{\alpha \mid x_{\alpha} \in \mathrm{B}[z, \varepsilon]\right\}$ is cofinal.

(iii) The net $\left(x_{\alpha}\right)_{\alpha}$ is a Cauchy net.

Then (iii) $\Rightarrow$ (i) $\Rightarrow$ (ii). And if $\left(x_{\alpha}\right)_{\alpha \in I}$ is universal, also (ii) $\Rightarrow$ (iii). (Hence, in the latter case, all three assertions are equivalent.)

Proof. (iii) $\Rightarrow$ (i): Let $\varepsilon>0$. Then there is $\alpha \in I$ such that $d\left(x_{\beta}, x_{\gamma}\right) \leq \varepsilon$ for all $\beta, \gamma \geq \alpha$. Hence $\mathrm{B}\left[\left\{x_{\alpha}\right\}, \varepsilon\right]$ contains the tail $\left\{x_{\beta} \mid \beta \geq \alpha\right\}$.

(i) $\Rightarrow$ (ii): Let $\varepsilon>\varepsilon^{\prime}>0$. Then there is a finite subset $F \subseteq K$ such that $K \subseteq$ $\bigcup_{z \in F} \mathrm{~B}\left(z, \varepsilon^{\prime}\right)$. Hence, by (ii), the set $\bigcup_{z \in F} \mathrm{~B}[z, 2 \varepsilon]$ contains a tail of $\left(x_{\alpha}\right)_{\alpha}$. Since $F$ is finite, there is $z \in F$ such that $\left\{\alpha \mid x_{\alpha} \in \mathrm{B}[z, 2 \varepsilon]\right\}$ is cofinal.

(ii) $\Rightarrow$ (iii): Suppose that $\left(x_{\alpha}\right)_{\alpha}$ is universal, let $\varepsilon>0$ and pick $z$ as in (ii). By the universality of the net either the set $A_{\varepsilon}:=\left\{\alpha \mid x_{\alpha} \in \mathrm{B}[z, \varepsilon]\right\}$ or its complement contains a tail of $I$. But since $A_{\varepsilon}$ is cofinal, the second alternative is impossible. Hence, $d\left(x_{\alpha}, x_{\beta}\right) \leq 2 \varepsilon$ for all $\alpha, \beta$ from a tail of $I$.

Theorem B.3. Let $\left(x_{\alpha}\right)_{\alpha \in I}$ be a net in a regular topological space $X$ and let

$$
C:=\bigcap_{\beta \in I} \overline{\left\{x_{\alpha} \mid \alpha \geq \beta\right\}}
$$

be its set of cluster points. Consider the following assertions.

(i) Each subnet of $\left(x_{\alpha}\right)_{\alpha \in I}$ has a cluster point.

(ii) Each universal subnet of $\left(x_{\alpha}\right)_{\alpha \in I}$ converges.

(iii) For each cofinal subsequence $\left(\alpha_{n}\right)_{n \in \mathbb{N}}$ the sequence $\left(x_{\alpha_{n}}\right)_{n \in \mathbb{N}}$ has a cluster point.

(iv) The set $C$ is non-empty and compact.

Then (i) $\Leftrightarrow$ (ii) $\Rightarrow$ (iii) and (i) $\Rightarrow$ (iv). If, in addition, I admits cofinal subsequences and $X$ is metrizable, then (iii) $\Rightarrow$ (iv) as well.

Proof. (i) $\Leftrightarrow$ (ii): This follows from Lemma B.1 and the fact that a universal net converges if and only if it has a cluster point.

(i) $\Rightarrow$ (iv): By hypothesis, the net $\left(x_{\alpha}\right)_{\alpha \in I}$ itself has a cluster point, so $C \neq \emptyset$. Let $\left(y_{j}\right)_{j \in J}$ be a universal net in $C$. It suffices to show that $\left(y_{j}\right)_{j}$ converges. Let

$$
M:=\left\{(\alpha, j, U) \mid \alpha \in I, U \text { open in } X \text { and } y_{k} \in U \text { for all } k \geq j\right\}
$$

be directed by

$$
\left(\alpha, j_{0}, U\right) \leq\left(\beta, j_{1}, V\right) \stackrel{\text { def. }}{\Longleftrightarrow} \alpha \leq \beta \wedge j_{0} \leq j_{1} \wedge V \subseteq U .
$$

For each $(\alpha, j, U) \in M$ one has $y_{j} \in U$ and hence there is

$$
\alpha \leq \varphi(\alpha, j, U) \in I \quad \text { with } \quad x_{\varphi(\alpha, j, U)} \in U .
$$

Then the mapping $\varphi: M \rightarrow I$ is cofinal, so $\left(x_{\varphi(\alpha, j, U)}\right)_{(\alpha, j, U) \in M}$ is a subnet. By hypothesis (i), this subnet has a cluster point $y \in X$, say. We prove that $y_{j} \rightarrow y$.

To this end, let $V$ be any open neighborhood of $y$ in $X$. Since $X$ is regular, there is an open set $W$ such that $y \in W \subseteq \bar{W} \subseteq V$. It suffices to show that $\bar{W}$ contains a tail of $\left(y_{j}\right)_{j}$. Suppose that this is not the case. Then, since that net is universal, the complement $U_{0}:=X \backslash \bar{W}$ contains tail of $\left(y_{j}\right)_{j}$. This means that there is $j_{0} \in J$ such that $y_{j} \in U_{0}$ for all $j \geq j_{0}$. In particular, $\left(\alpha, j_{0}, U_{0}\right) \in M$ for all $\alpha \in I$. 
Fix $\alpha_{0} \in I$. Then, since $y$ is a cluster point of $\left(x_{\varphi(m)}\right)_{m \in M}$ and $W$ is an open neighborhood of $y$, there is $M \ni(\alpha, j, U) \geq\left(\alpha_{0}, j_{0}, U_{0}\right)$ with $x_{\varphi(\alpha, j, U)} \in W$. But by the construction of $\varphi$ we have also $x_{\varphi(\alpha, j, U)} \in U \subseteq U_{0}$. Since $U_{0} \cap W=\emptyset$, this yields a contradiction and the proof is complete.

(i) $\Rightarrow$ (iii): This is trivial, as the sequence $\left(x_{\alpha_{n}}\right)_{n}$ is a subnet of $\left(x_{\alpha}\right)_{\alpha}$ whenever $\left(\alpha_{n}\right)_{n}$ is a cofinal sequence in $I$.

(iii) $\Rightarrow$ (iv): Suppose that $d$ is a metric inducing the topology of $X$ and that $I$ admits cofinal sequences. It then follows from (iii) that $C \neq \emptyset$. In order to see that $C$ is compact, let $\left(y_{n}\right)_{n}$ be a sequence in $C$ and let $\left(\alpha_{n}\right)_{n}$ be a cofinal sequence in $I$. Recursively, one can find a sequence $\left(x_{\beta_{n}}\right)_{n}$ such that $\beta_{n} \geq \alpha_{n}$ and $d\left(x_{\beta_{n}}, y_{n}\right) \leq \frac{1}{n}$. By (iii), $\left(x_{\beta_{n}}\right)_{n}$ has a cluster point. Since $X$ is metric, this means that $\left(x_{\beta_{n}}\right)_{n}$ has a convergent subsequence. But then $\left(y_{n}\right)_{n}$ also has convergent subsequence, and as $C$ is closed, the limit of this subsequence lies in $C$.

\section{Appendix C. Some Notions from Semigroup Theory}

A semigroup is a non-empty set $S$ together with an associative operation $S \times S \rightarrow S$, generically called "multiplication". A subset $M$ of a semigroup $S$ is multiplicative, if $M \cdot M \subseteq M$. A non-empty multiplicative subset is a subsemigroup. A non-empty subset $J$ of a semigroup $S$ is a (two-sided) ideal of $S$ if $S J \cup J S \subseteq J$. Each ideal is a subsemigroup.

A neutral element in a semigroup is any element $e \in S$ such that $e s=s e=s$ for all $s \in S$. There is at most one neutral element; if there is none, one can adjoin one in a standard way.

A semigroup $S$ is called Abelian or commutative if $s t=t s$ for all $s, t \in S$. It is common to write Abelian semigroups additively, and denote their neutral elements by 0 .

An Abelian semigroup $S$ is called cancellative if it satisfies the implication

$$
s+t=s+t^{\prime} \quad \Rightarrow \quad t=t^{\prime}
$$

for all $s, t, t^{\prime} \in S$. By a standard result from semigroup theory, $S$ is faithfully embeddable into a group if and only if $S$ is cancellative. In this case, one usually considers $S$ as a subset of some group $G$ such that $G=S-S$. The (necessarily Abelian) group $G$ is then unique up to canonical isomorphism and is called the group generated by $S$. In each case, when we speak of a group generated by an Abelian semigroup $S$, we have this meaning in mind, and in particular suppose tacitly that $S$ is cancellative.

An Abelian semigroup $S$ with neutral element 0 is called divisible, if for each $s \in S$ and $n \in \mathbb{N}$ there is $t \in S$ such that $n t=s$. (This definition extends the common notion of divisibility from groups to semigroups.) And $S$ is called essentially divisible if for each $s \in S$ and $n \in \mathbb{N}$ there are $t_{1}, t_{2} \in S$ such that $n t_{1}=s+n t_{2}$.

Each divisible semigroup and each semigroup that generates a divisible group is essentially divisible.

\section{REFERENCES}

[AB94] W. Arendt and A. V. Bukhvalov. Integral representations of resolvents and semigroups. Forum Math., 6(1):111-135, 1994.

[Are08] W. Arendt. Positive semigroups of kernel operators. Positivity, 12(1):25-44, 2008. 
[AS05] Y. Abramovich and G. Sirotkin. On order convergence of nets. Positivity, 9(3):287292, 2005.

[Axm80] D. Axmann. Struktur-und Ergodentheorie irreduzibler Operatoren auf Banachverbänden. PhD thesis, Eberhard-Karls-Universität zu Tübingen, 1980.

[Buk78] A. V. Bukhvalov. Integral representation of linear operators. Journal of Mathematical Sciences, 9(2):129-137, 1978.

[Dav05] E. B. Davies. Triviality of the peripheral point spectrum. J. Evol. Equ., 5(3):407-415, 2005.

[dJW14] M. de Jeu and M. Wortel. Compact groups of positive operators on Banach lattices. Indag. Math. (N.S.), 25(2):186-205, 2014.

[EFHN15] T. Eisner, B. Farkas, M. Haase, and R. Nagel. Operator Theoretic Aspects of Ergodic Theory, volume 272 of Graduate Texts in Mathematics. Springer, Cham, 2015.

[EKRW01] E. Yu. Emel'yanov, U. Kohler, F. Räbiger, and M. P. H. Wolff. Stability and almost periodicity of asymptotically dominated semigroups of positive operators. Proc. Amer. Math. Soc., 129(9):2633-2642, 2001.

[EN00] K.-J. Engel and R. Nagel. One-Parameter Semigroups for Linear Evolution Equations, volume 194 of Graduate Texts in Mathematics. Springer-Verlag, New York, 2000. With contributions by S. Brendle, M. Campiti, T. Hahn, G. Metafune, G. Nickel, D. Pallara, C. Perazzoli, A. Rhandi, S. Romanelli and R. Schnaubelt.

[Ger13] M. Gerlach. On the peripheral point spectrum and the asymptotic behavior of irreducible semigroups of Harris operators. Positivity, 17(3):875-898, 2013.

[Ger14] M. Gerlach. Semigroups of Kernel Operators. PhD thesis, Universität Ulm, 2014. DOI: 10.18725/OPARU-3263.

[GG17a] M. Gerlach and J. Glück. On a convergence theorem for semigroups of positive integral operators. C. R. Math. Acad. Sci. Paris, 355(9):973-976, 2017.

[GG17b] M. Gerlach and J. Glück. Convergence of positive operator semigroups. 2017. Preprint: arxiv.org/abs/1705.01587v1.

[GN82] G. Greiner and R. Nagel. La loi "zéro ou deux" et ses conséquences pour le comportement asymptotique des opérateurs positifs. J. Math. Pures Appl. (9), 61(3):261-273, 1982.

[Gre82] G. Greiner. Spektrum und Asymptotik stark stetiger Halbgruppen positiver Operatoren. Sitzungsber. Heidelb. Akad. Wiss. Math.-Natur. Kl., pages 55-80, 1982.

[GvE81] J. J. Grobler and P. van Eldik. A characterization of the band of kernel operators. Quaestiones Math., 4(2):89-107, 1980/81.

[Kei06] V. Keicher. On the peripheral spectrum of bounded positive semigroups on atomic Banach lattices. Arch. Math. (Basel), 87(4):359-367, 2006.

[Kun18] M. C. Kunze. Diffusion with nonlocal Dirichlet boundary conditions on unbounded domains. 2018. Preprint: arxiv.org/abs/1810.04474v1.

[LZ71] W. A. J. Luxemburg and A. C. Zaanen. Riesz spaces. Volume I. North-Holland Publishing Co., Amsterdam-London; American Elsevier Publishing Co., New York, 1971. North-Holland Mathematical Library.

[MN91] P. Meyer-Nieberg. Banach Lattices. Universitext. Springer-Verlag, Berlin, 1991.

[PR00] K. Pichór and R. Rudnicki. Continuous Markov semigroups and stability of transport equations. J. Math. Anal. Appl., 249(2):668-685, 2000.

[PR16] K. Pichór and R. Rudnicki. Asymptotic decomposition of substochastic operators and semigroups. J. Math. Anal. Appl., 436(1):305-321, 2016.

[PR18] K. Pichór and R. Rudnicki. Asymptotic decomposition of substochastic semigroups and applications. Stoch. Dyn., 18(1):1850001, 18, 2018.

[Sch71] E. Scheffold. Das Spektrum von Verbandsoperatoren in Banachverbänden. Math. Z., 123:177-190, 1971.

[Sch74] H. H. Schaefer. Banach Lattices and Positive Operators. Springer-Verlag, New York, 1974. Die Grundlehren der mathematischen Wissenschaften, Band 215.

[Wnu99] W. Wnuk. Banach Lattices with Order Continuous Norms. Warsaw: Polish Scientific Publishers PWN, 1999.

[Wol08] M. P. H. Wolff. Triviality of the peripheral point spectrum of positive semigroups on atomic Banach lattices. Positivity, 12(1):185-192, 2008.

[Zaa83] A. C. Zaanen. Riesz spaces II, volume 30 of North-Holland Mathematical Library. North-Holland Publishing Co., Amsterdam, 1983. 
Ulm University, Institute of Applied Analysis, Helmholtzstr. 18, 89081 Ulm, GerMANY

E-mail address: jochen.glueck@alumni.uni-ulm.de

Kiel University, Mathematisches Seminar, Ludewig-Meyn-Str.4, 42118 Kiel, Germany

E-mail address: haase@math.uni-kiel.de 